\title{
The Frequency-dependent Damping of Slow Magnetoacoustic Waves in a Sunspot Umbral Atmosphere
}

\author{
S. Krishna Prasad ${ }^{1}$ (10, D. B. Jess ${ }^{1,2}$ (1), T. Van Doorsselaere ${ }^{3}$ (1) , G. Verth ${ }^{4}$ (i), R. J. Morton ${ }^{5}$ (10), \\ V. Fedun ${ }^{6}$, R. Erdélyi ${ }^{7,8}$ (1) and D. J. Christian ${ }^{2}$ (1) \\ ${ }^{1}$ Astrophysics Research Centre, School of Mathematics and Physics, Queen's University Belfast, Belfast, BT7 1NN, UK; krishna.prasad@qub.ac.uk \\ ${ }^{2}$ Department of Physics and Astronomy, California State University Northridge, Northridge, CA 91330, USA \\ ${ }^{3}$ Centre for mathematical Plasma Astrophysics, Mathematics Department, KU Leuven, Celestijnenlaan 200B bus 2400, B-3001 Leuven, Belgium \\ ${ }^{4}$ School of Mathematics and Statistics, The University of Sheffield, Hicks Building, Hounsfield Road, Sheffield, S3 7RH, UK \\ ${ }^{5}$ Department of Mathematics, Physics and Electrical Engineering, Northumbria University, Ellison Building, Newcastle upon Tyne, NE1 8ST, UK \\ ${ }^{6}$ Department of Automatic Control and Systems Engineering, University of Sheffield, Sheffield, S1 3JD, UK \\ ${ }^{7}$ Solar Physics \& Space Plasma Research Centre (SP2RC), School of Mathematics and Statistics, University of Sheffield, Sheffield S3 7RH, UK \\ ${ }^{8}$ Department of Astronomy, Eötvös Loránd University, Budapest, P.O. Box 32, H-1518, Hungary \\ Received 2017 May 29; revised 2017 July 19; accepted 2017 August 14; published 2017 September 14
}

\begin{abstract}
High spatial and temporal resolution images of a sunspot, obtained simultaneously in multiple optical and UV wavelengths, are employed to study the propagation and damping characteristics of slow magnetoacoustic waves up to transition region heights. Power spectra are generated from intensity oscillations in sunspot umbra, across multiple atmospheric heights, for frequencies up to a few hundred $\mathrm{mHz}$. It is observed that the power spectra display a power-law dependence over the entire frequency range, with a significant enhancement around $5.5 \mathrm{mHz}$ found for the chromospheric channels. The phase difference spectra reveal a cutoff frequency near $3 \mathrm{mHz}$, up to which the oscillations are evanescent, while those with higher frequencies propagate upward. The power-law index appears to increase with atmospheric height. Also, shorter damping lengths are observed for oscillations with higher frequencies suggesting frequency-dependent damping. Using the relative amplitudes of the $5.5 \mathrm{mHz}$ (3 minute) oscillations, we estimate the energy flux at different heights, which seems to decay gradually from the photosphere, in agreement with recent numerical simulations. Furthermore, a comparison of power spectra across the umbral radius highlights an enhancement of high-frequency waves near the umbral center, which does not seem to be related to magnetic field inclination angle effects.
\end{abstract}

Key words: magnetohydrodynamics (MHD) - methods: observational - Sun: atmosphere - Sun: oscillations sunspots

\section{Introduction}

Slow magnetoacoustic waves (SMAWs) are, in general, compressive in nature, which makes them easily detectable through imaging observations. With the advent of highresolution observations from ground- and space-based instruments, both standing and propagating SMAWs have been discovered in a multitude of structures in the solar atmosphere including magnetic pores (Morton et al. 2011; Dorotovič et al. 2014; Moreels et al. 2015; Freij et al. 2016), chromospheric network (Vecchio et al. 2007; Kontogiannis et al. 2010, 2014), coronal loops (Wang et al. 2015; Jess et al. 2016), and polar plumes (Krishna Prasad et al. 2011; Su 2014). Recent multiwavelength observations of sunspots reveal that umbral flashes (Beckers \& Tallant 1969; Rouppe van der Voort et al. 2003) and running penumbral waves (Giovanelli 1972; Bloomfield et al. 2007; Freij et al. 2014) observed in the chromosphere, and quasi-periodic propagating disturbances observed in the coronal loops (Berghmans \& Clette 1999; De Moortel et al. 2000; De Moortel 2009), are just different manifestations of the SMAWs that propagate from the photosphere through to the corona (Jess et al. 2012a; Krishna Prasad et al. 2015; Zhao et al. 2016). It has been proposed that the interaction of photospheric $p$-modes with magnetic fields generates different magnetohydrodynamic (MHD) waves (Spruit 1991; Cally et al. 1994; Jess et al. 2015), of which SMAWs are guided upward along the magnetic field lines (Cally 2007; Khomenko \& Cally 2012), allowing them to reach coronal heights
(De Pontieu et al. 2003, 2005). Furthermore, it has been shown that oscillations with frequencies below the typical acoustic cutoff can also be channeled into the corona if the magnetic fields are inclined (De Pontieu et al. 2005; Erdélyi 2006), or if the radiative losses are included (Khomenko et al. 2008).

The available energy flux of SMAWs in the corona is on the order of a few hundred, to a few thousand erg cm $\mathrm{cm}^{-2} \mathrm{~s}^{-1}$ (McEwan $\&$ de Moortel 2006), which is not at all sufficient to maintain the corona at million-kelvin temperatures $\left(\sim 10^{7} \mathrm{erg} \mathrm{cm}^{-2} \mathrm{~s}^{-1}\right.$; Withbroe \& Noyes 1977). Nevertheless, the photospheric acoustic oscillations, particularly those at high frequencies, were thought to possess enough energy to replenish the chromospheric radiative losses. However, using the power spectra from quiet-Sun intensity fluctuations and one-dimensional numerical simulations, Fossum \& Carlsson (2005) have shown that the acoustic energy flux found in the $5-50 \mathrm{mHz}$ frequency range is substantially smaller than expected; about one-tenth of that required in the chromosphere. The authors employed data from the Transition Region and Coronal Explorer (TRACE; Handy et al. 1999) in the $1700 \AA$ and $1600 \AA$ passbands to arrive at this conclusion. In contrast, three-dimensional numerical simulations by Wedemeyer-Böhm et al. (2007) indicate the existence of sufficient energy flux from acoustic waves. Furthermore, Wedemeyer-Böhm et al. (2007) demonstrate that the limited spatial resolution of TRACE observations underestimate the acoustic power by at least an order of magnitude, helping to explain the discrepancy. Subsequent observations with higher resolution have revealed 

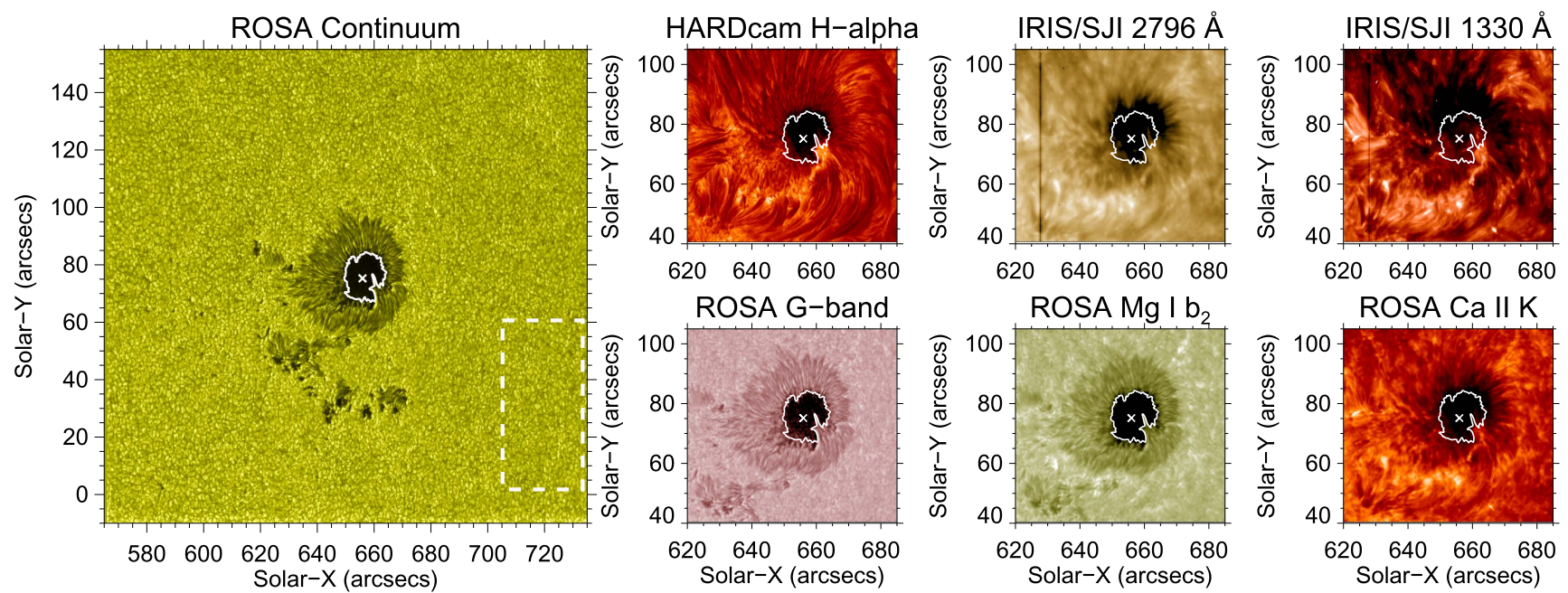

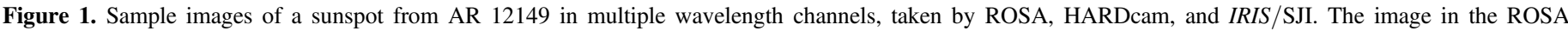

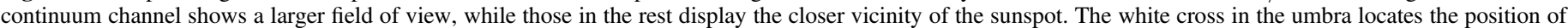

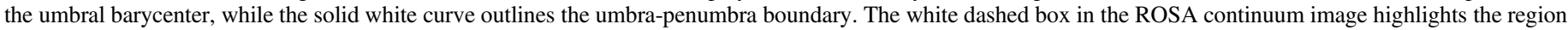

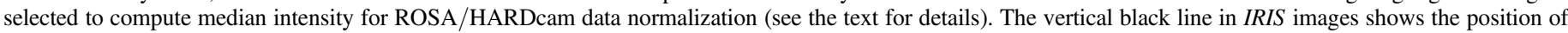
the IRIS slit outside the umbra.

larger, but still either insufficient (Carlsson et al. 2007) or barely comparable (Bello González et al. 2009, 2010) acoustic power required to dominate chromospheric heating. One must note, however, that the chromosphere, being magnetically rich with expanding magnetic fields, supports different MHD wave modes that are both compressible and incompressible (e.g., Morton et al. 2012; Kuridze et al. 2013; Jess et al. 2017), which may make a significant contribution to localized heating.

In magnetized atmospheres, the energy contribution of acoustic oscillations (through SMAWs) to chromospheric heating is relatively less studied. Two-dimensional numerical simulations of small-scale network fields driven by transverse impulses reveal insufficient acoustic flux to balance chromospheric radiative losses (Vigeesh et al. 2009). Furthermore two- and threedimensional MHD simulations by Fedun et al. (2011) and Vigeesh et al. (2012) indicate variable acoustic energy flux in the upper atmospheric layers depending on the choice of the driver (see also Mumford et al. 2015; Mumford \& Erdélyi 2015). In a sunspot umbra, using the root-mean-squared (rms) velocities of $\mathrm{NaI} \mathrm{D}_{1}$ and $\mathrm{Na} \mathrm{I}_{2}$ lines, Kneer et al. (1981) estimate an outward energy flux of $5 \times 10^{4} \mathrm{erg} \mathrm{cm}^{-2} \mathrm{~s}^{-1}$, which is well below the chromospheric requirement of $2.6 \times 10^{6} \mathrm{erg} \mathrm{cm}^{-2} \mathrm{~s}^{-1}$ (Avrett 1981). In a recent study, Felipe et al. (2011) computed the acoustic energy flux as a function of atmospheric height using data-driven MHD simulations of a sunspot umbra. The authors found insufficient energy $\left(\approx 10^{6} \mathrm{erg} \mathrm{cm}^{-2} \mathrm{~s}^{-1}\right)$ even at the lowermost (photospheric) height investigated, which further decreases with height. Chae et al. (2017) calculate the average energy flux in three-minute oscillations over a sunspot umbra, observed in the NiI $5436 \AA$ line that forms $38 \mathrm{~km}$ above photosphere, as $1.8 \times 10^{6} \mathrm{erg} \mathrm{cm}^{-2} \mathrm{~s}^{-1}$. This value is on the same order of that obtained by Felipe et al. (2011) near the photosphere. On the contrary, based on the observational data of a sunspot umbra, Kanoh et al. (2016) estimate an energy flux of $2 \times 10^{7} \mathrm{erg} \mathrm{cm}^{-2} \mathrm{~s}^{-1}$ at the photospheric level, with $8.3 \times 10^{4} \mathrm{erg} \mathrm{cm}^{-2} \mathrm{~s}^{-1}$ at the lower transition region level in the 6-10 $\mathrm{mHz}$ frequency band, implying a dissipation of sufficient energy to maintain the umbral chromosphere. The authors, however, add a caveat that the energies could be overestimated due to opacity effects.
In this article, we utilize high-resolution, high-cadence image sequences obtained simultaneously in multiple wavelengths, to study the damping of SMAWs in a sunspot umbra up to transition region heights. Representative power spectra of the sunspot umbra are generated across all channels to perform this in-depth study. We present the observational aspects of the data in Section 2, followed by our analysis and results in Section 3, and finally discuss our important interpretations in Section 4.

\section{Observations}

The Dunn Solar Telescope (DST), situated in the Sacramento Peak mountains of New Mexico, was employed to obtain highresolution images of active region NOAA 12149 at a very high cadence in five different wavelength channels using the Rapid Oscillations in the Solar Atmosphere (ROSA; Jess et al. 2010) and the Hydrogen-Alpha Rapid Dynamics camera (HARDcam; Jess et al. 2012a). The observations were carried out on 2014 August 30 starting from 14:37 UT for approximately 3 hrs. Four identical ROSA cameras were used to capture images simultaneously in four wavelength channels corresponding to the blue continuum (4170 ̊), G-band (4305.5 $)$, Mg I b 2 (5172.7 $)$, and Ca II K line core (3933.7 $\AA$ ), while the HARDcam instrument acquired images in the $\mathrm{H} \alpha$ line core $(6562.8 \AA)$. The bandpass widths of each of these channels are $52 \AA, 9.2 \AA, 0.13 \AA, 1 \AA$, and $0.25 \AA$, respectively. All of the images were processed following standard procedures. In addition to the application of high-order adaptive optics (Rimmele 2004) during the observations, all of the data were subjected to speckle reconstruction algorithms (Wöger et al. 2008) to improve the image quality. The final cadences of the data are $2.11 \mathrm{~s}$ for the blue continuum, G-band and $\mathrm{CaII} \mathrm{K}, 4.22 \mathrm{~s}$ for the $\mathrm{MgIb}_{2}$, and $0.99 \mathrm{~s}$ for the $\mathrm{H} \alpha$ channels.

The data were subsequently normalized with a median intensity value obtained from a relatively quiet region (outlined by a white dashed box in Figure 1) in the field of view to compensate for brightness changes over time that result from changes in the elevation of the Sun. The atmospheric seeing conditions remained excellent throughout the time series, barring a few short-term local fluctuations that affected a 
Table 1

Typical Formation Heights of Different ROSA/HARDcam Channels above the Photosphere

\begin{tabular}{|c|c|}
\hline Channel Name & Formation Height \\
\hline Blue continuum $(4170 \AA)$ & $25 \mathrm{~km}(1)$ \\
\hline G-band & 100 km (1) \\
\hline $\operatorname{Mg~I~} b_{2}$ & 700 km (2) \\
\hline Ca II K & 1300 km (3) \\
\hline $\mathrm{H} \alpha$ & 1500 km (4) \\
\hline
\end{tabular}

References. (1) Jess et al. (2012b), (2) Schmieder (1979), (3) Beebe \& Johnson (1969), (4) Vernazza et al. (1981).

relatively small number of images in each channel. These images were identified through the decreases in their contrast ratios with time, and were subsequently replaced through interpolation. Data from different channels were coaligned with respect to the blue continuum using a set of calibration images that were obtained immediately prior to the science observations. The spatial sampling of the ROSA data is 0 !" 18 per pixel. HARDcam $(\mathrm{H} \alpha)$ images are observed at a better spatial resolution $\left(0 . \prime 09\right.$ pixel $^{-1}$; as a result of the four-fold increase in pixel numbers), but for the analyses presented in this article, these data have been resampled to match the ROSA plate scale. The typical formation heights of individual ROSA and HARDcam channels are listed in Table 1.

The Interface Region Imaging Spectrograph (IRIS; De Pontieu et al. 2014) also observed AR 12149 during the same timeframe. IRIS observations consist of sit-and-stare data lasting approximately $6 \mathrm{hr}$, commencing from 11:12 UT and lasting until 17:13 UT. The corresponding images taken by the slit-jaw imager (SJI) were obtained only in two channels, $2796 \AA$ and $1330 \AA$. The spectrographic slit was pointed entirely outside the sunspot, therefore providing no associated spectra for the umbra under investigation. Thus, we consider only the SJI data, between 14:13 and 17:13 UT, which has the maximum temporal overlap with the ROSA/HARDcam image sequences. The $2796 \AA$ and $1330 \AA$ channels mainly capture the plasma present in the upper chromosphere and the transition region, respectively (De Pontieu et al. 2014). All of the data are processed to "level 2" standard, which incorporates dark current subtraction, flat-fielding, and other necessary corrections, including the images being brought on to a common plate scale. Additionally, we also ensured that each time sequence is properly coaligned with the first image using intensity crosscorrelations. The cadence of the data is approximately $18.8 \mathrm{~s}$ and the spatial sampling is $\approx 00^{\prime \prime} 166$ per pixel. Robust coalignment between the ROSA, HARDcam, and IRIS channels has been achieved by cross-correlating the ROSA Ca II K and IRIS $2796 \AA$ images. Sample images for each of the channels are displayed in Figure 1. The ROSA blue continuum image displays the full ground-based field of view, while the rest display zoom-ins of the primary sunspot captured in each of the respective channels.

\section{Analysis and Results}

Active region NOAA 12149 has a single, large, nearly circular sunspot of negative polarity (Hale class $\beta / \beta$ ), surrounded by a few pore-like magnetic concentrations (of the same polarity) and some diffuse positive flux. The detailed structure of the sunspot and its vicinity across different ROSA,
HARDcam, and IRIS channels can be seen in Figure 1. Using the time-averaged blue continuum image, a boundary between the umbra and penumbra of the sunspot has been defined through intensity thresholding, calculated in relation to the median granulation intensity from the immediate surroundings. The location of the umbral barycenter in intensity is then computed from the pixel locations within this outer perimeter following the methods detailed by Jess et al. (2013). The computed umbra-penumbra boundary and the umbral barycenter are marked by a white solid line and a white cross, respectively, in Figure 1. The excellent coalignment between different optical and UV channels may also be noted.

\subsection{Mean Power Spectra}

A Fourier power spectrum of a time series reveals the oscillation frequencies manifesting within it as individual spikes in power. Time series from each pixel location within the umbra was subjected to Fourier analysis using the Fast Fourier Transform (FFT) method, with the corresponding frequencies, along with their respective power peaks, identified and noted. In addition to performing FFT analyses on a pixelby-pixel basis, we also calculated a global average Fourier power spectrum for all pixels contained within the umbral perimeter using the bootstrap method (Efron 1979).

The mean power spectra calculated following this method for all ROSA and HARDcam data are displayed in Figure 2, along with the respective $1 \sigma$ uncertainties. As may be noted, the uncertainties are fairly small, smaller than the size of the symbol in most cases. The corresponding plots for the IRIS $2796 \AA$ and $1330 \AA$ channels are shown in Figure 3 . Note that the highest frequency up to which the power spectra are plotted is different for each of the ROSA, HARDcam, and IRIS channels due to differences in cadences of the respective data sets. The power in all channels decreases with frequency (implying a power-law dependence), albeit with an intermediate bump visible in some channels and a plateau of power at higher frequencies.

The flattening of power at higher frequencies is due to the white noise that dominates the signal at those frequencies. Assuming enough photon statistics ${ }^{9}$ in the umbra, the white noise is primarily composed of photon noise. The photon noise, by nature, follows a Poisson distribution, and has an amplitude proportional to the square root of the signal. To estimate the level of this noise present in the data, for each umbral pixel, we generate a random light curve following a Poisson distribution, with an amplitude equivalent to the square root of the mean intensity at that pixel. Using these artificially generated noise light curves, we compute the mean power spectrum in an identical way to that of the original data. Hence, the modeled noise power spectra, for each channel after appropriate scaling, are shown as red dots in Figures 2 and 3.

The computed noise levels are subtracted from the individual power spectra, with the resultant power spectra for all ROSA, HARDcam, and IRIS channels displayed in Figures 4 and 5. In these figures, the vertical bars in gray represent the corresponding uncertainties, which are substantially larger at high frequencies due to the lower power values obtained after white noise subtraction. Also, the errors appear asymmetric due to the $\log$-scale employed. It may be noted that the applied correction

\footnotetext{
9 For example, the signal is well above the detector background noise characteristics such as the dark noise etc.
} 

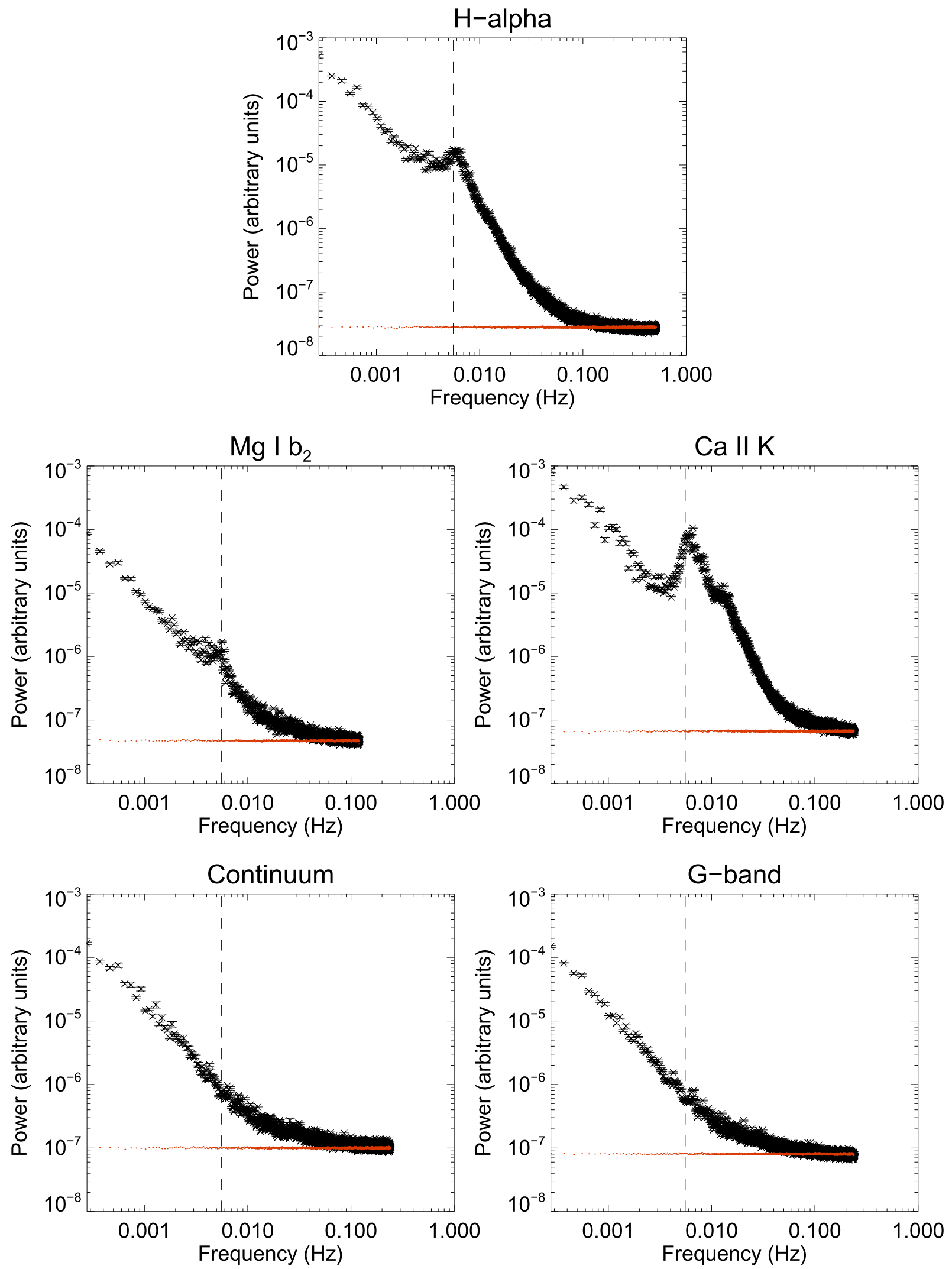

Figure 2. Mean power spectra generated from intensity oscillations within the umbra, across multiple ROSA/HARDcam channels. The corresponding $1 \sigma$ uncertainties on the values are shown as error bars, which are on the order of the size of the symbol. The red dots display the level of photon noise present in the data, computed from artificially generated light curves that follow a Poisson distribution, with amplitudes equivalent to the square root of the mean observed intensity. The vertical dashed line denotes a frequency of $5.5 \mathrm{mHz}$, where significant enhancements in power can be seen in chromospheric channels. Note that the frequency range of the spectra differs in each of the channels due to differences in their respective cadences. 

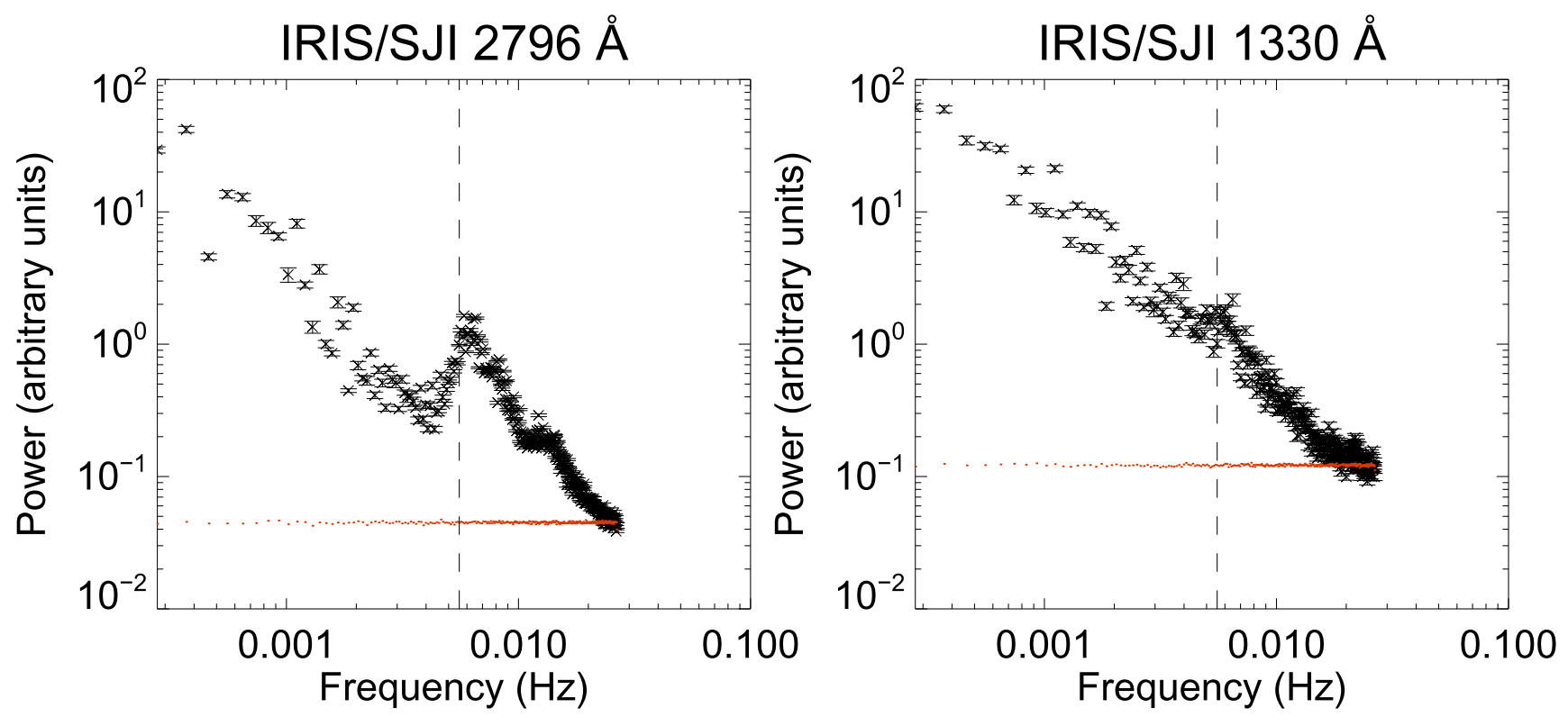

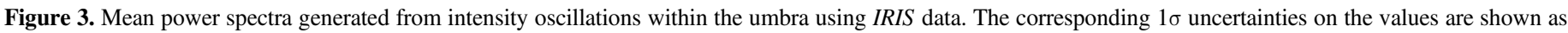

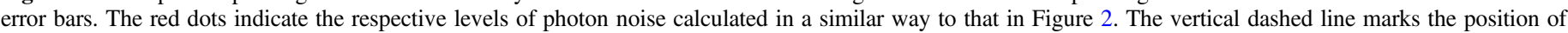
$5.5 \mathrm{mHz}$. Note that the extent of the spectra is lower than that in ROSA/HARDcam data (Figure 2) due to the lower temporal cadence of the IRIS data.

for white noise is similar to the methods adopted by Fossum \& Carlsson (2005) and Lawrence et al. (2011). The original power spectra are also shown in these plots, in gray, for comparison. It seems that, following the subtraction of white noise, the same power-law dependence continues to be present at higher frequencies. In order to quantify the exact dependence, each power spectrum, in a logarithmic scale, was fitted linearly following least-squares minimization (Markwardt 2009). Red solid lines in Figures 4 and 5 indicate the best fits to the data. The corresponding slopes, which represent the power-law indices in a linear scale, are also listed in each plot. The data in the intermediate peaks, between 3.3 and $50 \mathrm{mHz}(300-20 \mathrm{~s}$; denoted by vertical dotted lines in the figure) are ignored while fitting the power spectra for ROSA and HARDcam channels. This range is restricted to $3.3-16.6 \mathrm{mHz}(300-60 \mathrm{~s})$ for IRIS data, due to their lower cadence. Also, since there is a substantially larger number of data points at high frequencies (right-hand side of the peak) than those at the low frequencies (left-hand side of the peak), the least-squares fit would be strongly biased toward the highfrequency data, which is not desirable. In order to avoid this, we used differential weights ${ }^{10}$ for the low- and the highfrequency data while fitting. The resultant fitted slopes, as shown in Figures 4 and 5, appear to increase with solar atmospheric height.

The intermediate bumps in the power spectra peak near $5.5 \mathrm{mHz}(\approx 180 \mathrm{~s}$; marked by a vertical dashed line in Figures 2 and 3), which is a characteristic frequency of SMAWs often observed in the umbral chromosphere. The photospheric umbra usually displays oscillations at a frequency of $\approx 3.3 \mathrm{mHz}$. Umbral oscillations at these frequencies are believed to be connected to the photospheric $p$-modes (e.g., Krishna Prasad et al. 2015; Zhao et al. 2016). Several theories also exist to explain the transition of the characteristic frequency from

\footnotetext{
${ }^{10}$ The weights for all the low-frequency data $(<3.3 \mathrm{mHz})$ were set to unity and for all the high-frequency data $(>50 \mathrm{mHz}$ for ROSA and $>16.6 \mathrm{mHz}$ for IRIS data) were set to 0.1 , while those for the intermediate frequencies were set to zero.
}

$3.3 \mathrm{mHz}$ in the photosphere to $5.5 \mathrm{mHz}$ in the chromosphere (Zhugzhda et al. 1983; Fleck \& Schmitz 1991). However, oscillation amplitudes in the photosphere are usually very low, which are further minimized by the opacity effects present in intensity measurements (e.g., Khomenko \& Collados 2015). This is perhaps the reason why no bumps are observed in the blue continuum and G-band power spectra. It may be noted that the level of enhancement in the bump is variable across the different imaging channels. This is due to the varying amplitude of oscillations as the waves propagate through different atmospheric layers. However, a direct comparison of the power in different channels is not particularly trivial since it depends on several physical and instrumental factors, including the spectral region, exposure time, filter bandwidth, etc. Therefore, the power spectra in each channel are normalized to their respective power-law fits, which provides a relative indication of the amplitude of oscillations present in that atmospheric layer, and thus may be safely compared across different channels to gain additional physical insight.

Figure 6 displays the normalized power spectra for various ROSA, HARDcam, and IRIS channels. Different colors and symbols are used to denote the data from different channels. The uncertainties on these data are similar to those displayed in Figures 4 and 5, but are not shown here to avoid cluttering from larger values at high frequencies. The vertical dotted lines mark the locations of $3.3 \mathrm{mHz}(300 \mathrm{~s})$ and $50 \mathrm{mHz}(20 \mathrm{~s})$. As can be seen, there is no obvious peak in the blue continuum and G-band channels. The power present in the rest of the channels peaks at $\approx 6.57 \mathrm{mHz}(152 \mathrm{~s}),{ }^{11}$ barring the $\mathrm{Mg} \mathrm{I} \mathrm{b}_{2}$ channel, which peaks at $\approx 5.65 \mathrm{mHz}(177 \mathrm{~s})$. Besides the main peak, two additional peaks are visible in the ROSA Ca II K and IRIS $2796 \AA$ channels at $\approx 8.3 \mathrm{mHz}(120 \mathrm{~s})$ and $\approx 13.1 \mathrm{mHz}$ (76 s) frequencies, of which the latter is more prominent.

The peak power appears to increase from $\mathrm{Mg} \mathrm{I}_{2}$ to $\mathrm{Ca} \mathrm{II} \mathrm{K}$, before further decreasing in the neighboring $\mathrm{H} \alpha$ and IRIS channels. This implies an increase and decrease of the

\footnotetext{
${ }^{11}$ In IRIS $1330 \AA$ channel, the power peaks at $6.48 \mathrm{mHz}(154 \mathrm{~s})$.
} 

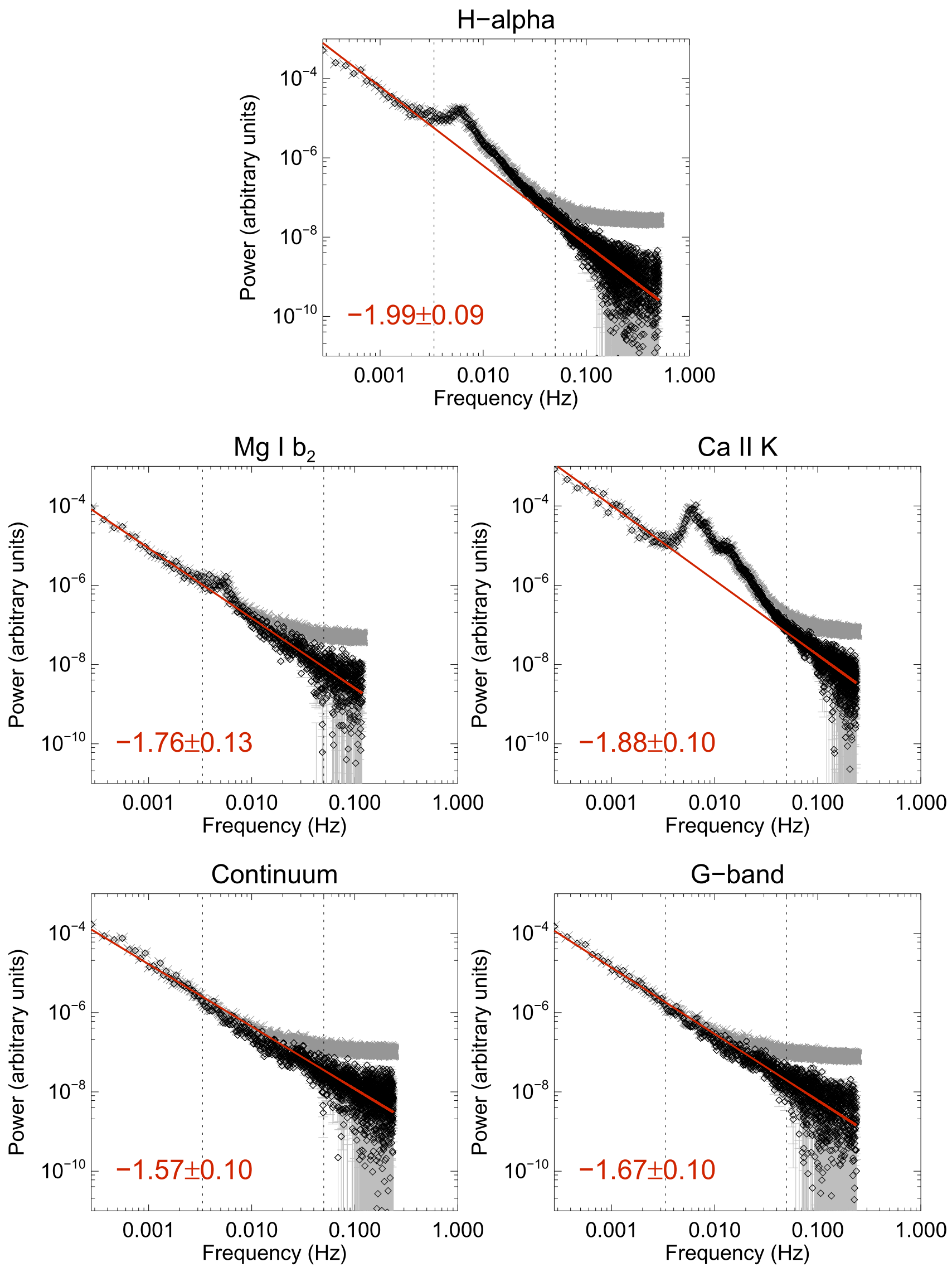

Figure 4. Resultant power spectra in ROSA/HARDcam channels, following the subtraction of photon noise from each of the mean power spectra. The vertical bars in gray represent the corresponding uncertainties. The original mean power spectra are also shown in gray for comparison. Red solid lines represent the best linear fits to the data using a logarithmic scale, highlighting a clear power-law dependence. The respective power-law indices, as obtained from the slopes, are also listed in each plot. The data between the vertical dashed lines, positioned at 3.3 and $50 \mathrm{mHz}$, are ignored while performing the linear fits. 

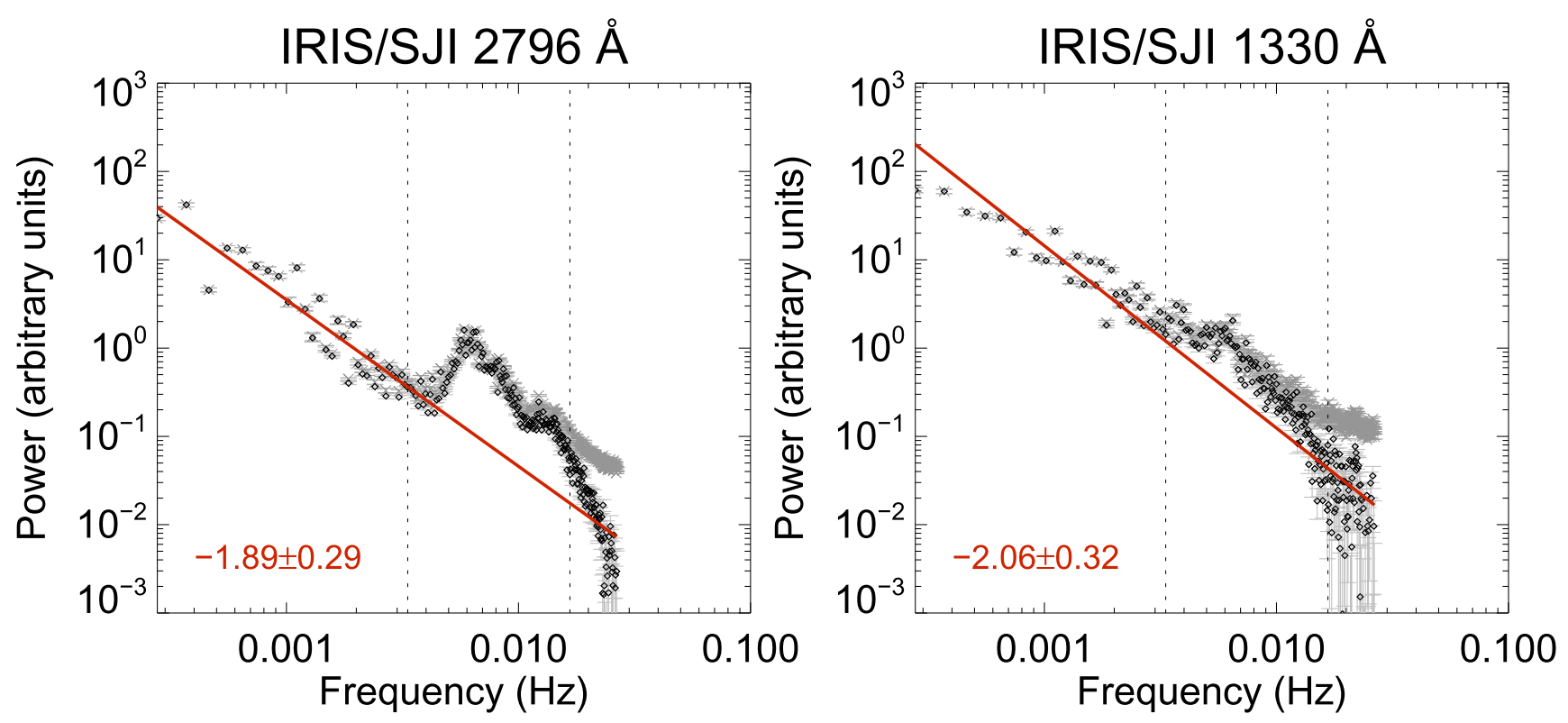

Figure 5. Same as Figure 4, but here for IRIS data. The data between 3.3 and $16.6 \mathrm{mHz}$ are ignored during fitting, as denoted by the vertical dashed lines.

oscillation amplitudes across these channels. Considering the square root of peak power in each channel as a measure of oscillation amplitude, we plot the variation of amplitude with atmospheric height in the left panel of Figure 7. Colors and symbols used to represent data from different channels, are the same as that in Figure 6. The corresponding uncertainties are shown as vertical bars plotted adjacent to the data for clarity. The power at $5.55 \mathrm{mHz}(\approx 180 \mathrm{~s})$ is used to calculate the blue continuum and G-band amplitudes. As listed in Table 1, the formation heights chosen for the blue continuum, G-band, $\mathrm{Mg} \mathrm{I} \mathrm{b}_{2}$, Ca II K, and $\mathrm{H} \alpha$ are $25 \mathrm{~km}$ (Jess et al. 2012b), $100 \mathrm{~km}$ (Jess et al. 2012b), $700 \mathrm{~km}$ (Schmieder 1979), $1300 \mathrm{~km}$ (Beebe \& Johnson 1969), and $1500 \mathrm{~km}$ (Vernazza et al. 1981) above the photosphere, respectively. The IRIS $2796 \AA$ channel, with a bandpass of $4 \AA$, is sensitive to the plasma present in the upper chromosphere, while the $1330 \AA$ channel, with a bandpass of $55 \AA$, is sensitive to transition region plasma (De Pontieu et al. 2014). This is also evident from the structures visible in the IRIS images (Figure 1). Taking the close resemblance between $\mathrm{Ca}$ II $\mathrm{K}$ and IRIS $2796 \AA$ images into account, the formation height of the $2796 \AA$ channel is chosen as $1400 \mathrm{~km}$, while the $1330 \AA$ channel is deemed to be representative of $2000 \mathrm{~km}$ above the photosphere (consistent with typical transition region heights). Alternative formation heights that may be possible for the $\mathrm{H} \alpha$ and IRIS $1330 \AA$ channels, as inferred from the phase difference spectra (see Section 3.2), are used to mark the gray diamond and square, respectively, in Figure 7. The corresponding errors are shown on the left-hand side for these data. We must emphasize that the plotted amplitudes obtained from the normalized power do not in any way constitute absolute values, but instead provide a representative comparison between the strengths of the oscillations observed at different atmospheric heights.

We also computed the energy flux $(F)$ following WKB approximation, using $F=\rho\left\langle\delta v^{2}\right\rangle c_{s}$, where $\rho, \delta v$, and $c_{s}$, are the mass density, velocity amplitude, and sound speed respectively. The sound speed $c_{s}$ is related to the temperature $T$, as $c_{s}=\sqrt{\gamma R T / \mu}$, where $\gamma$ is the polytropic index, $R$ is the gas constant, and $\mu$ is the mean molecular weight. We consider $\gamma=5 / 3, R=8.314 \times 10^{7} \mathrm{erg} \mathrm{K}^{-1} \mathrm{~mol}^{-1}$, and $\mu=0.61$ (Mariska 1993), in these calculations. The required temperature and density values corresponding to the observational formation heights were acquired through spline interpolation of the values extracted from the umbral core "M" model of Maltby et al. (1986). The velocity amplitudes are obtained by scaling the ROSA blue continuum Fourier amplitude to $40 \mathrm{~m} \mathrm{~s}^{-1}$. This corresponds to the rms velocity amplitude (integrated in the $5-8 \mathrm{mHz}$ band) averaged over two sunspot umbrae observed by Lites \& Thomas (1985) in the Ti I $6304 \AA$ line that forms $\approx 40 \mathrm{~km}$ above the photosphere (Abdelatif et al. 1984), close to the formation height of the ROSA blue continuum channel. Furthermore, from a compilation of similar results from several authors it is shown that the rms oscillatory amplitudes in the $5-8 \mathrm{mHz}(2-3$ minutes) band in the umbral photosphere falls within the range of 25-50 $\mathrm{m} \mathrm{s}^{-1}$ (Table 1; Thomas \& Weiss 1992). Also, more recently, Chae et al. (2017) report similar amplitudes $\left(33 \mathrm{~m} \mathrm{~s}^{-1}\right)$ for three-minute oscillations over a sunspot umbra using observations in the Ni I $5436 \AA$ line that forms $\approx 38 \mathrm{~km}$ above the photosphere. The axis on the right-hand side of the left panel in Figure 7 shows the scaled amplitudes in $\mathrm{m} \mathrm{s}^{-1}$. The computed energy flux values are shown in the right panel of Figure 7, with an additional axis on the right to show the energy flux in S.I. units $\left(\mathrm{W} \mathrm{m}^{-2}\right)$. The gray diamond and square indicate the energy flux in the $\mathrm{H} \alpha$ and IRIS $1330 \AA$ channels, respectively, assuming alternative formation heights for the data based on phase difference analysis (see Section 3.2). The error bars adjacent to the data represent the corresponding uncertainties. As can be seen, the energy flux gradually decreases, even when the oscillation amplitude is increasing, suggesting the damping of SMAWs across these atmospheric layers. It may be noted that modifying the velocity amplitudes in the photosphere will change the energy flux by an order of magnitude or more. However, the decreasing trend in energy flux, where our main emphasis lies, remains unchanged.

SMAWs have been reported to undergo frequency-dependent damping in the solar corona (Ofman \& Wang 2002; Krishna Prasad et al. 2014; Gupta 2014). In order to explore if such behavior is also exhibited by these waves in the 


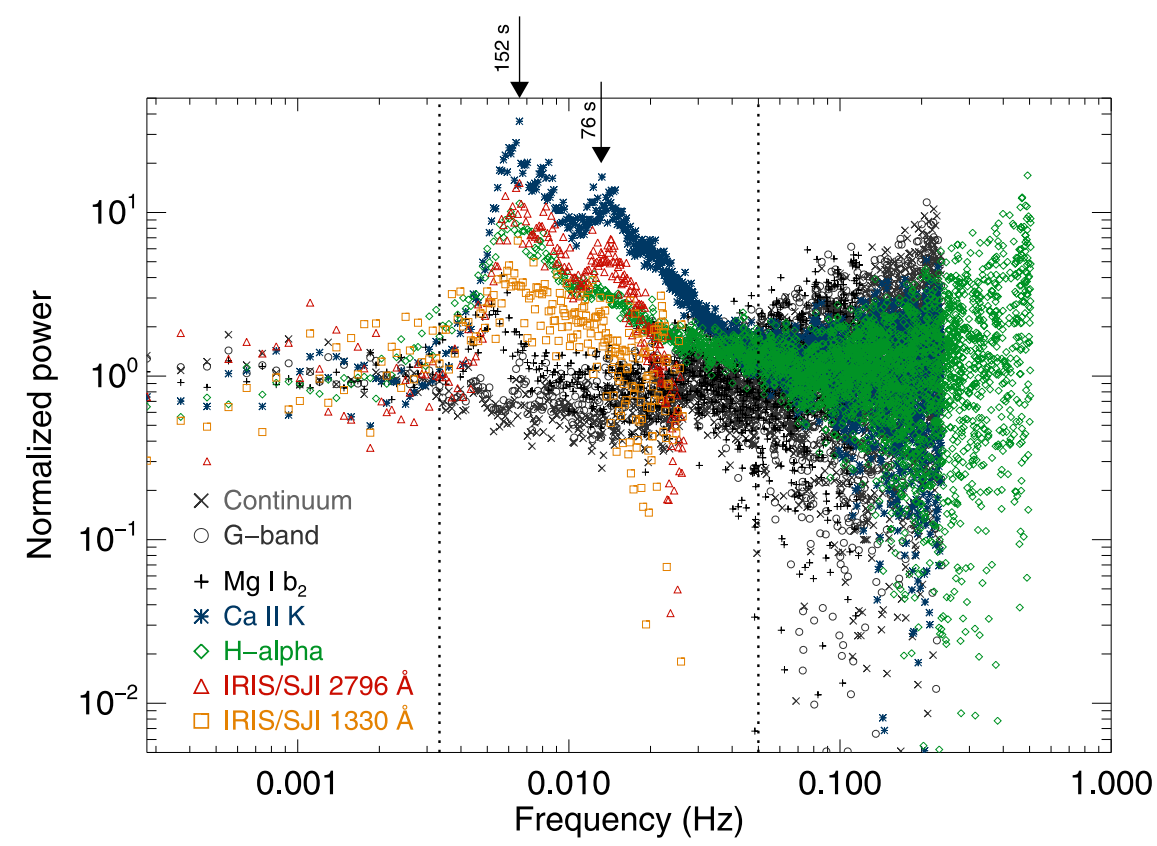

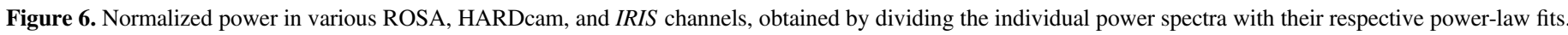

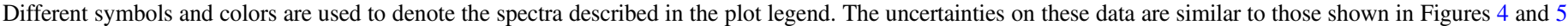

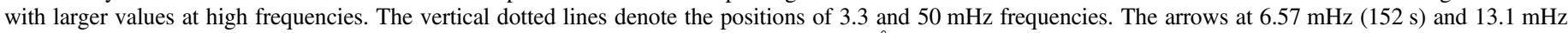

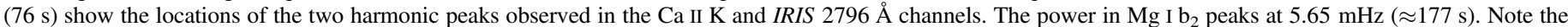
different strengths of the peaks found in different channels, which indicates varying oscillation amplitudes.

sub-coronal layers, we computed oscillation amplitudes at three frequencies coincident with the identified peaks at $6.57,8.33$, and $13.1 \mathrm{mHz}$. The original power spectra (after correction for white noise) were used to calculate the amplitudes to avoid potential influences of the varying power-law slopes on the frequency dependence. The individual power spectra were, however, normalized with their respective power at $3 \mathrm{mHz}$ to enable comparison across different channels. The power integrated in a $1 \mathrm{mHz}$ band around each of the frequency peaks $(0.5 \mathrm{mHz}$ on either side of the peak) is used to evaluate the amplitudes. The data in Ca II K, IRIS $2796 \AA$, $\mathrm{H} \alpha$, and IRIS $1330 \AA$ channels synonymous with the declining phase of amplitudes, are only considered. Figure 8 displays the computed amplitudes as a function of formation height of the channels along with the respective uncertainties. Solid lines represent the best fits to the data following a function $A=A_{0} e^{-\frac{h}{L_{d}}}+C$, where $A$ is the oscillation amplitude, $h$ is the formation height, $L_{d}$ is the damping length, and $A_{0}$ and $C$ are appropriate constants. The exponential decay function appears to be consistent with the data. Damping lengths obtained from the fits, at each frequency, $f$, are listed in the plot, which indicate frequency-dependent damping of the waves with stronger damping found at higher frequencies.

\subsection{Phase Difference Spectra}

The phase difference between oscillations at different heights can be used to determine whether the waves are upward/ downward propagating or standing (e.g., Centeno et al. 2006). We computed phase differences at all frequencies between the channel pairs ( $\mathrm{Mg} \mathrm{I}_{2}, \mathrm{Ca}$ II K), ( $\left.\mathrm{Mg} \mathrm{I}_{2}, \mathrm{H} \alpha\right),(\mathrm{Ca} \mathrm{II} \mathrm{K}, \mathrm{H} \alpha)$, and (IRIS $2796 \AA, 1330 \AA$ ), using their respective cross-power spectra. Data from individual pixel locations were directly matched in these calculations, assuming negligible magnetic field expansion between the formation heights of channels in each pair. This assumption is perhaps reasonable within a sunspot umbra where the magnetic field is mostly vertical. Also, the $\mathrm{Ca} I \mathrm{~K}$ and $\mathrm{H} \alpha$ data were resampled to match the lower temporal cadence of the $\mathrm{Mg} \mathrm{I} \mathrm{b}_{2}$ channel, which made the comparison between these channels more straightforward.

The resulting phase difference spectra, incorporating the data from the entire umbra, are plotted in Figure 9 for all of the channel pairs. At each frequency, the phase difference values across the umbral pixels are grouped into histograms that constitute the color scale employed in each plot. Therefore, the color only indicates the predominance of one value over the other, with red/yellow representing more frequent and blue/ green representing less frequent values. These diagrams are similar to those obtained by Felipe et al. (2010, 2011). The phase difference appears to repeat cyclically between $-\pi$ and $+\pi$ due to the $2 \pi$ indetermination in the computed phases previously noted by Centeno et al. (2006). The values are plotted only up to $50 \mathrm{mHz}$ as the photon noise dominates over the signal beyond this range (see Figure 2). Note that the IRIS data are plotted only up to $\approx 27 \mathrm{mHz}$ due to their lower cadence. Following Centeno et al. (2006), we generated a theoretical phase difference curve (yellow solid lines in Figure 9) for each channel pair. Wave propagation in a stratified isothermal atmosphere with radiative cooling (Centeno et al. 2006) is considered. However, to accommodate for the varying temperatures along the wave propagation path, phase differences are calculated in steps of $100 \mathrm{~km}$ (within which the isothermal approximation is employed) between the estimated formation heights of the respective channels, which are then integrated over the full path to get the final phase difference curves for a channel pair. The required temperature values at each step are obtained from the umbral core "M" model of Maltby et al. (1986) through spline interpolation. The radiative cooling time is kept constant at $45 \mathrm{~s}, 20 \mathrm{~s}, 15 \mathrm{~s}$, and $10 \mathrm{~s}$ for the channel pairs $\left(\mathrm{MgIb}_{2}, \mathrm{CaIIK}\right),\left(\mathrm{Mg} \mathrm{I}_{2}, \mathrm{H} \alpha\right)$, 

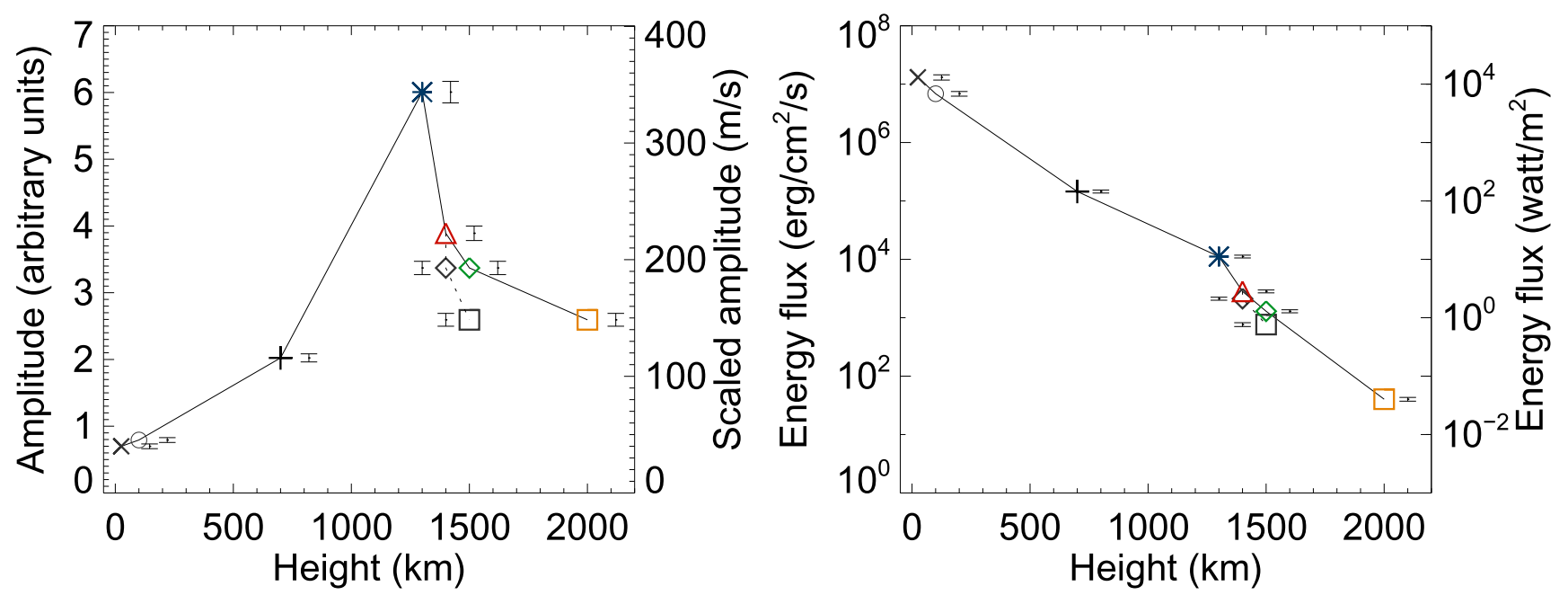

Figure 7. Left: relative amplitudes of $5.5 \mathrm{mHz}$ (three-minute) oscillations at different heights, derived from the normalized peak power in various ROSA, HARDcam, and IRIS channels. Different colors and symbols are used to represent different channels following the same notation as in Figure 6. The gray diamond and square represent the data from the $\mathrm{H} \alpha$ and IRIS $1330 \AA$ channels, respectively, assuming alternative formation heights, as obtained from the phase difference spectra (see Section 3.2 for details). The axis on the right shows the amplitude values after scaling the blue continuum Fourier amplitudes to $40 \mathrm{~m} \mathrm{~s}^{-1}$. Right: corresponding energy flux obtained by using temperatures and densities from the Maltby et al. (1986) umbral " $M$ " sunspot model. The axis on the right shows the same energy flux values in S.I. units. Respective uncertainties on the amplitude and the energy flux values are shown as vertical bars, plotted adjacent to the data for clarity.

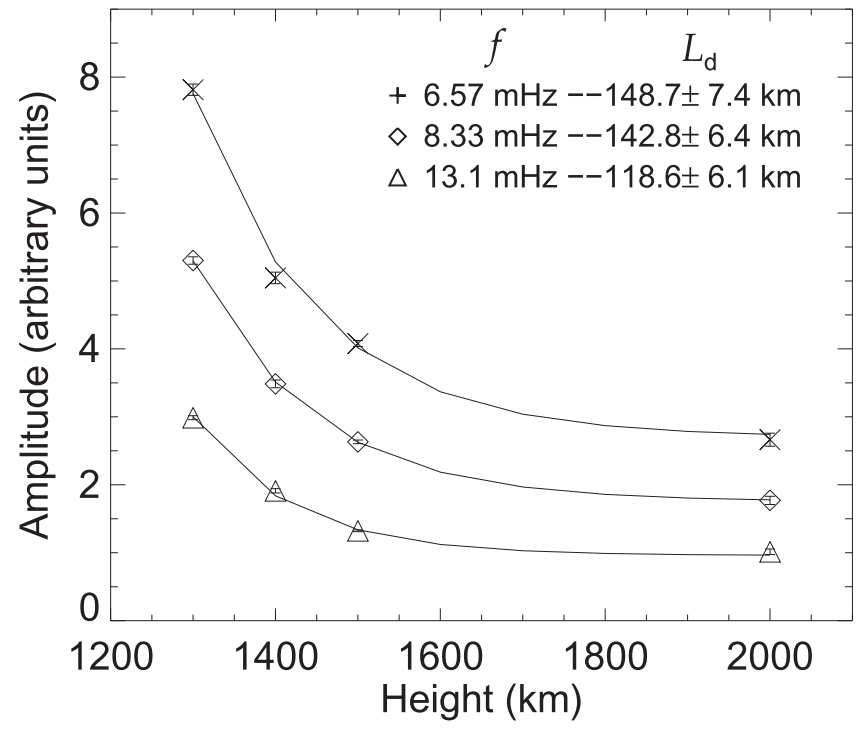

Figure 8. Oscillation amplitudes, along with the corresponding uncertainties, at three different frequencies obtained from the original power spectra for $\mathrm{Ca}$ II $\mathrm{K}$, IRIS $2796 \AA, \mathrm{H} \alpha$, and IRIS $1330 \AA$ channels. Each of the spectra were normalized with their respective power at $3 \mathrm{mHz}$ to enable comparison across multiple channels. The power integrated over a $1 \mathrm{mHz}$ band chosen around each frequency, is used to compute the amplitudes. Solid lines represent exponential fits to the data. The damping lengths $\left(L_{d}\right)$ obtained from the fitted values along with the respective central frequencies $(f)$ are listed in the plot.

(Ca II K, H $\alpha$ ), and (IRIS $2796 \AA, 1330 \AA$ ), respectively. These values are approximately on the same order of those used in previous calculations (Centeno et al. 2006; Bloomfield et al. 2007; Felipe et al. 2010).

As can be seen from the phase difference spectra for the $\left(\mathrm{Mg} \mathrm{I}_{2}, \mathrm{Ca}\right.$ II $\left.\mathrm{K}\right)$ and $\left(\mathrm{Mg} \mathrm{I}_{2}, \mathrm{H} \alpha\right)$ pairs, the phase difference initially stays near zero up to about $3 \mathrm{mHz}$ (marked by a white dashed line in Figure 9), before increasing linearly beyond. This implies that oscillations with frequencies above $3 \mathrm{mHz}$ are propagating upward, while those below are evanescent between the two layers. Previous studies on sunspot umbrae have shown a similar cutoff frequency at about $4 \mathrm{mHz}$ (Centeno et al. 2006; Felipe et al. 2010), but as demonstrated by Centeno et al. (2009), the exact value depends on the physical conditions of the structure. Theoretical phase difference curves are computed assuming the formation heights of $700 \mathrm{~km}$ for $\mathrm{Mg} \mathrm{I}_{2}, 1300 \mathrm{~km}$ for $\mathrm{Ca}$ II K, and $1500 \mathrm{~km}$ for $\mathrm{H} \alpha$ channels (see Table 1). The theoretical curves show a reasonably good fit until about $13 \mathrm{mHz}$, beyond which the observed phase differences appear noisy. It must be noted that the phase difference spectra represents values from individual pixel locations, unlike the power spectra shown in Figures 2-5 that display averages across the entire sunspot umbra. This perhaps explains why the phase differences at higher frequencies appear noisier, even though the oscillation power is well above the background noise level. This could also be partly related to the lower cadence of the $\mathrm{Mg} \mathrm{I}_{2}$ observations, resulting in more significant photon noise at relatively lower frequencies compared to $\mathrm{Ca}$ II $\mathrm{K}$ and $\mathrm{H} \alpha$ channels (see Figure 4).

The phase difference spectra for the (Ca II K, $\mathrm{H} \alpha$ ) and (IRIS $2796 \AA$, $1330 \AA$ ) pairs are shown in the top two panels of Figure 9. Here, the theoretical phase difference curves assume formation heights of $1300 \mathrm{~km}, 1400 \mathrm{~km}, 1500 \mathrm{~km}$, and $2000 \mathrm{~km}$ for the Ca II K, IRIS $2796 \AA$, H $\alpha$ and IRIS $1330 \AA$ channels, respectively, which are shown as white dotted lines. Note that the oscillation amplitudes in these channels are fairly high when compared to those found in the $\mathrm{Mg}$ I $\mathrm{b}_{2}$ observations (see Figures 6 and 7), which makes these phase difference spectra reliable up to frequencies as high as $13 \mathrm{mHz}$, and possibly beyond. Hence, the significant departure of the observed phase differences from the theoretical values might indicate the assumed formation heights are wrong. Indeed, the yellow solid lines, which seem to agree well with the observations, correspond to a formation height difference of $<100 \mathrm{~km}$ between each channel. Nevertheless, the positive phase difference values, combined with the already inferred upward propagation between $\mathrm{Mg} \mathrm{I} \mathrm{b}_{2}$ and $\mathrm{H} \alpha$ heights, implies a higher formation height for $\mathrm{H} \alpha$ and IRIS $1330 \AA$ when compared to Ca II K and IRIS $2796 \AA$ A, respectively. While we acknowledge the fact that it is not trivial to assign a single formation height to any of these channels, the remarkable 


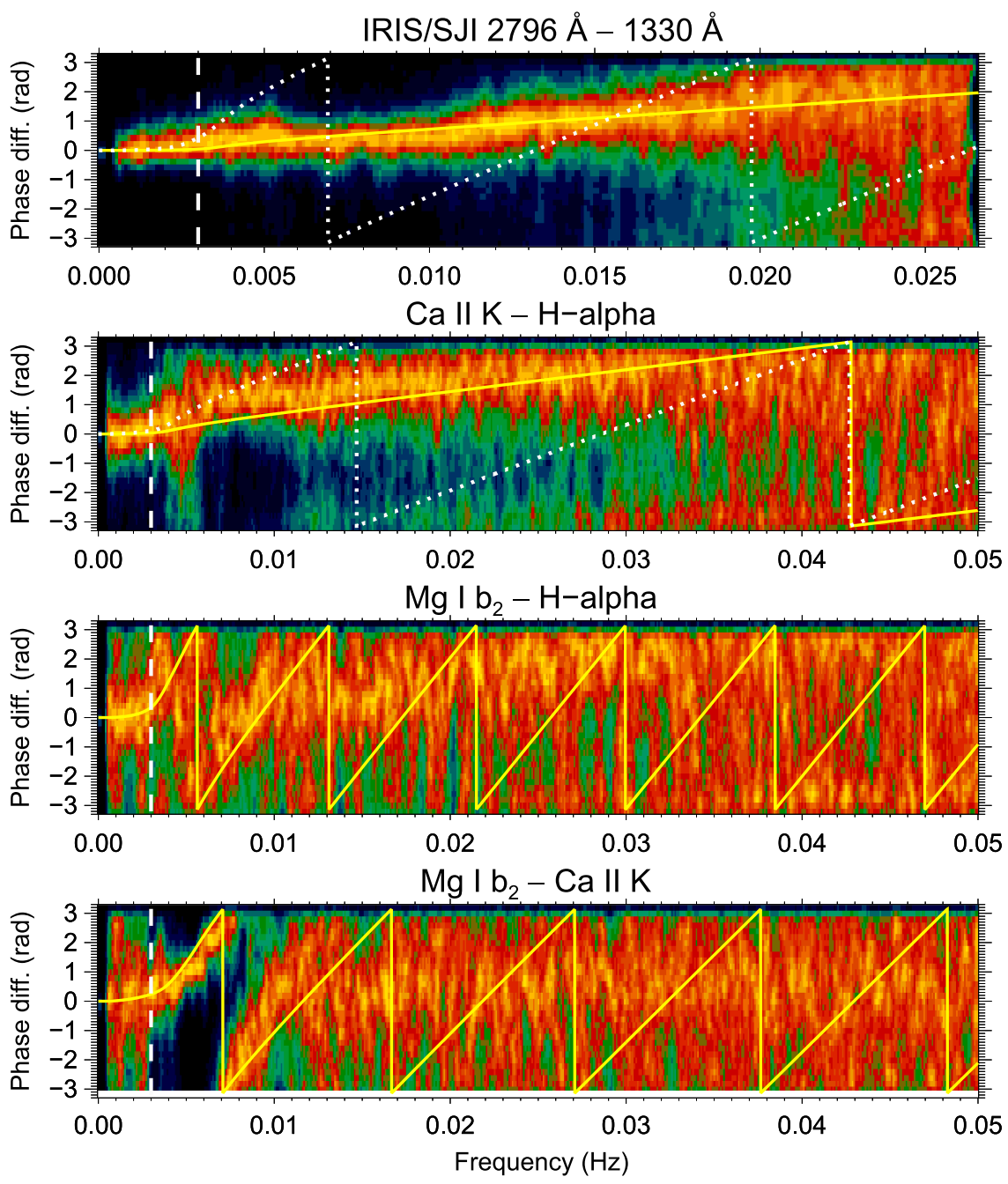

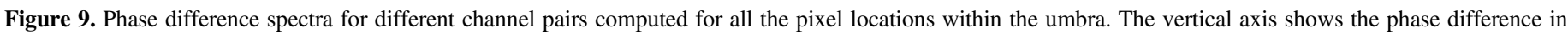

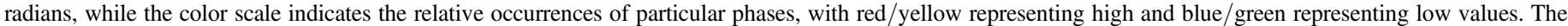

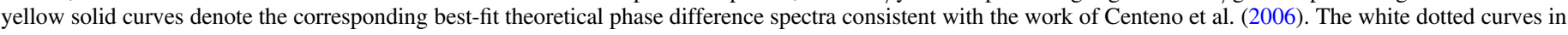

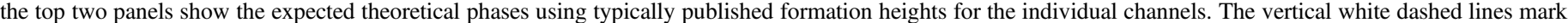
the positions of the $3 \mathrm{mHz}$ frequency, which distinguishes evanescent and propagating waves.

differences in the visible structures of the sunspot, between the $\mathrm{Ca}$ II K and $\mathrm{H} \alpha$ channels and between the IRIS $2796 \AA$ and $1330 \AA$ channels, suggest that the difference in formation heights could be larger than $100 \mathrm{~km}$. However, the relatively broad filter bandpasses of the Ca II K and IRIS SJI channels may also contribute to these uncertainties, since different regions of the solar atmosphere may dominate their respective contribution functions in drastically different ways. Indeed, it is possible that the observed formation height differences may be much smaller within the umbra, where the plasma is inherently cooler with significantly reduced opacities, when compared to other plage and quiet-Sun locations. On the other hand, SMAWs propagating above the $\mathrm{Ca}$ II $\mathrm{K}$ height may be predominantly nonlinear, and since the phase speed of nonlinear waves is larger than their linear counterparts, the difference in formation heights obtained from the linear wave theory may be substantially underestimated (Felipe et al. 2010). Also, one must note that the phase difference spectra generated from the intensity oscillations alone is often difficult to interpret, unlike those from the velocity oscillations, since the phase relation between intensity and velocity is a complex function of frequency and radiative losses
(Mihalas \& Mihalas 1984; Deubner \& Fleck 1989), which perhaps could partially explain the discrepancy.

\subsection{Radial Variation}

In Section 3.1, we addressed the mean power spectra computed from all pixel locations within the umbra. However, it is important to know whether the power spectra remain the same throughout the entire umbra. In order to study this, we implement the methods presented by Jess et al. (2016) and generate mean power spectra from a series of expanding annuli centered on the umbral barycenter. The width of each annulus is kept at 1 pixel and the radius is varied from 0 to the farthest point on the umbra-penumbra boundary. A mean power spectrum is calculated from all pixel locations falling within an annulus following the same procedures described in Section 3.1. For the outer annuli, any pixel locations falling outside the umbral penumbral boundary are ignored in the subsequent calculations.

The obtained power spectra for the ROSA/HARDcam and IRIS data are shown in Figures 10 and 11, respectively. The 


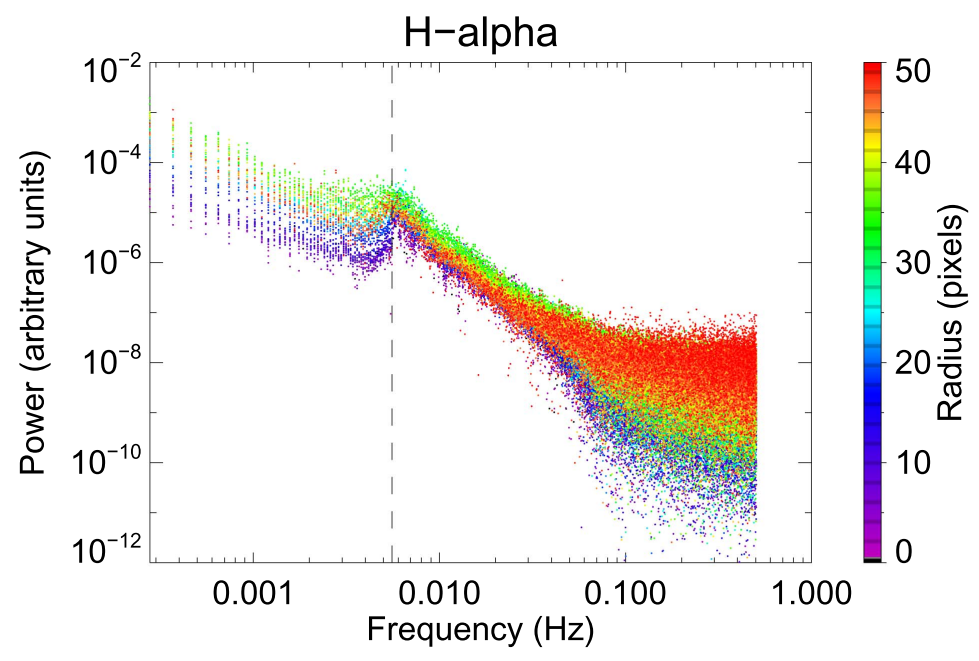

$\mathrm{Mg} \mid \mathrm{b}_{2}$

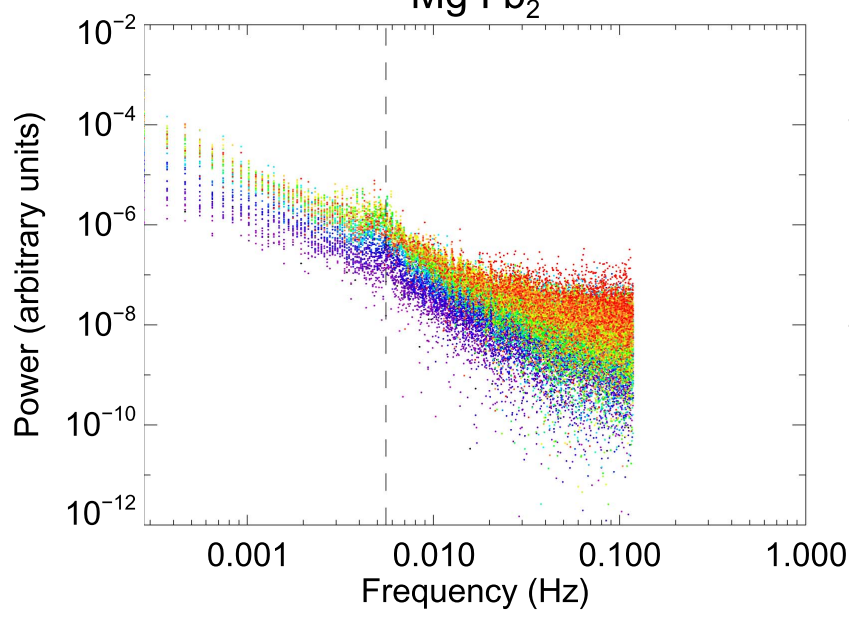

Continuum

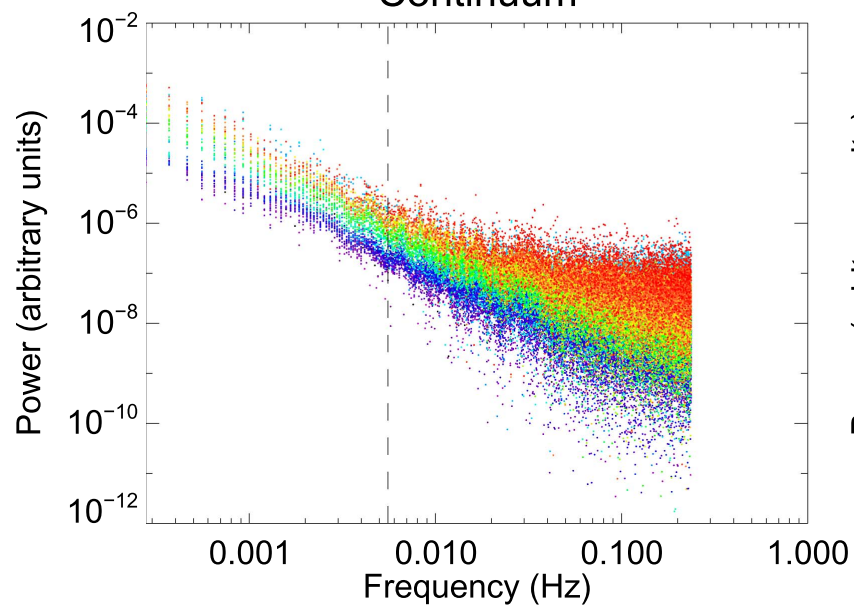

Ca II K
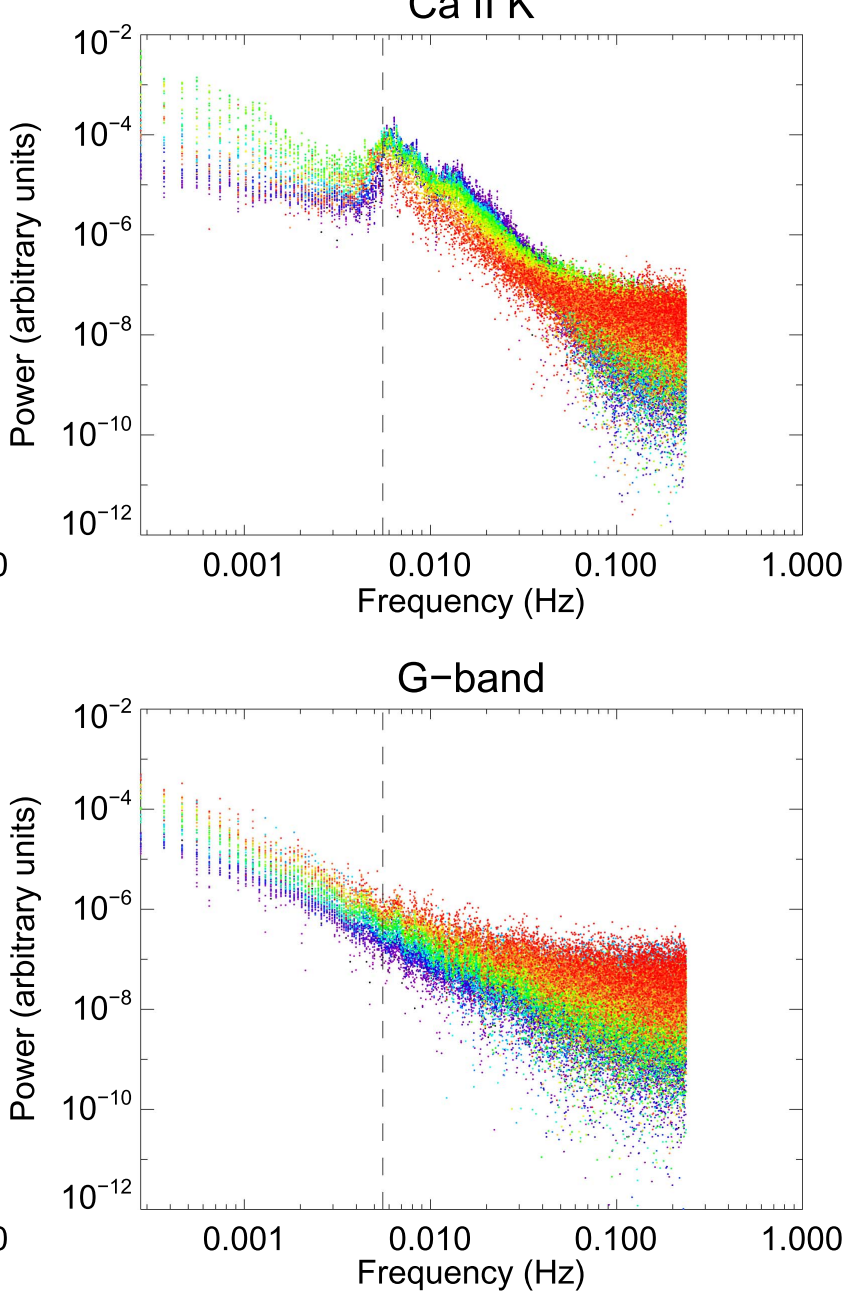

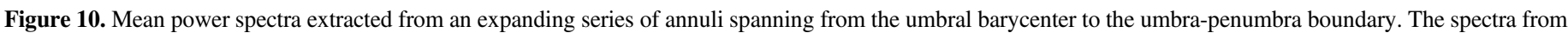

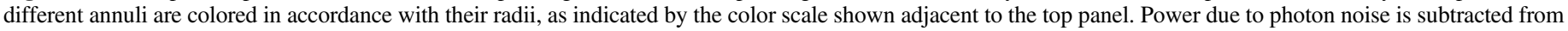
the individual spectra before plotting. The vertical dashed line marks the position of $5.5 \mathrm{mHz}$ where strong chromospheric peaks are identified.

color scale in each of these plots documents the radius of the annulus (in pixels) from which the power spectra is generated. Note that the maximum radius in the IRIS data is slightly larger due to its finer spatial sampling. As described in Section 3.1, the corresponding photon noise power has been calculated and subtracted from the individual spectra before plotting. Some flattening is still evident at high frequencies, which may be a result of limited number statistics pertaining to the 1-pixel wide annuli employed. Nevertheless, the overall frequency dependence appears to be roughly the same as in Figures 4 and 5. The 

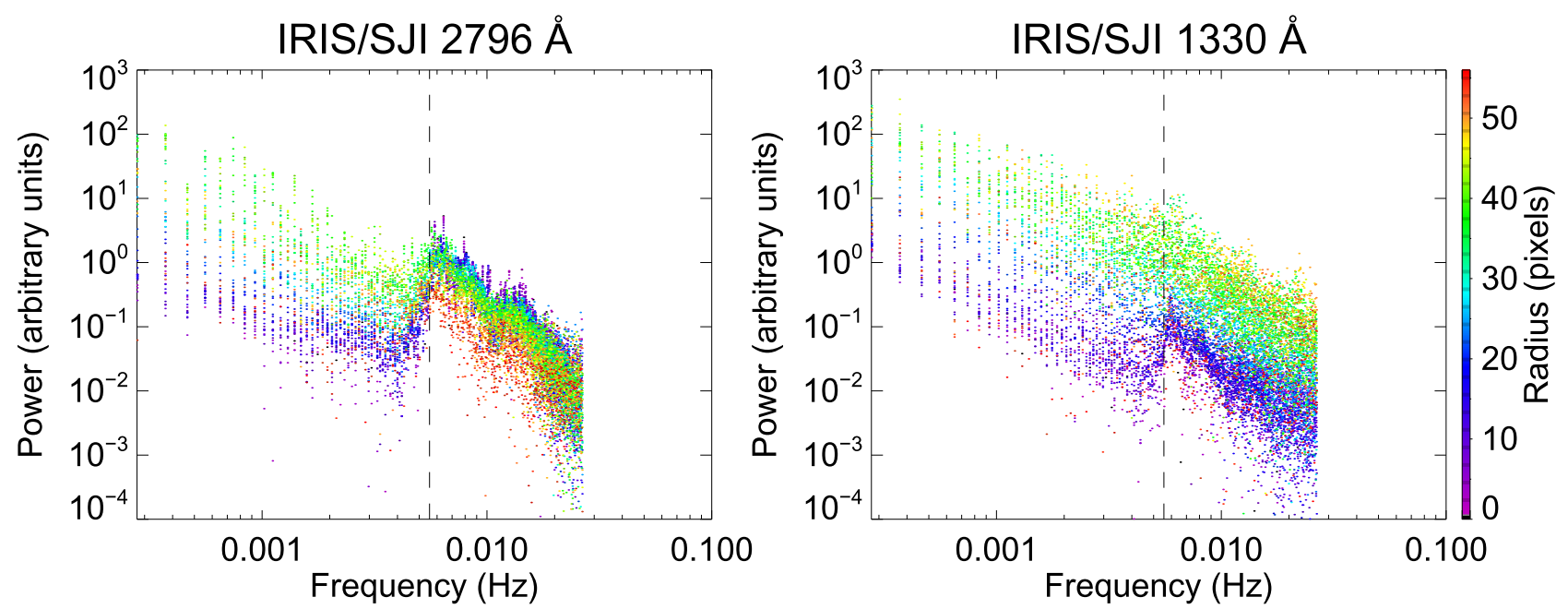

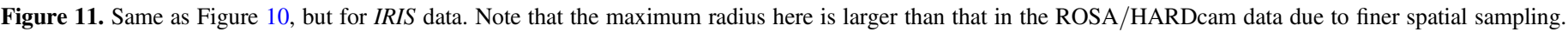

corresponding uncertainties are also similar but not shown in these plots, to avoid cluttering at high frequencies. As shown, the power falls with increasing frequency following a powerlaw relationship in all channels, including a visible enhancement near $5.5 \mathrm{mHz}$ (dashed line in Figures 10 and 11) in all but the photospheric blue continuum and G-band channels. In general, there is larger power at distances farther from the umbral barycenter across all frequencies. An exception is the power around $5.5 \mathrm{mHz}$ in the $\mathrm{Ca}$ II K, $\mathrm{H} \alpha$, and IRIS $2796 \AA$ channels, where the power/distance behavior appears to be reversed, i.e., the power near the umbral center is higher than that at the outer radii. This is perhaps similar to the findings of Reznikova et al. (2012), where the authors show that oscillations with relatively higher frequencies are more pronounced near the umbral center than at the peripheral regions. Raja Bayanna et al. (2014) reported similar observations suggesting larger power at high frequencies in a sunspot umbra compared to that at the umbra-penumbra boundary at chromospheric heights. Reznikova et al. (2012) interpreted this behavior as being due to the differences in inclinations of the magnetic fields across the umbra. However, as one may notice, the $\mathrm{MgI} \mathrm{b}_{2}$ channel does not show any such enhancement in the power closer to the umbral center, and even more surprisingly, the IRIS $1330 \AA$ Aransition region channel does not show this either. If it has to do with magnetic field inclinations, one would expect this effect to be at least present, if not more pronounced, in the IRIS $1330 \AA$ channel that captures plasma at more elevated atmospheric heights. This abnormal enhancement near the umbral center at purely chromospheric heights is perhaps caused by a different physical mechanism.

\section{Discussion and Conclusions}

Power spectra of a sunspot umbra were generated and studied at multiple atmospheric heights using simultaneous intensity images captured by the ROSA and HARDcam instruments at the DST and by IRIS/SJI. The spectra could be generated up to frequencies as high as a few hundred $\mathrm{mHz}$ thanks to the ultra-high-cadence observations provided by ROSA/HARDcam. A power-law dependence of the oscillation power is found across the entire frequency range and at all heights, with significant power enhancements found near $5.5 \mathrm{mHz}$ in chromospheric channels. Gabriel et al. (1997) observed a similar dependence, with an enhancement at $3 \mathrm{mHz}$ found in the power spectrum generated from the line-of-sight velocity oscillations of integrated solar disk light. While the peak at $3 \mathrm{mHz}$ is due to photospheric $p$-modes, the authors describe the power-law dependence at lower frequencies as being due to the random velocity fields introduced by solar convection. However, in the present case, convection is expected to be suppressed in the sunspot umbra, meaning the existence of a similar dependancy at chromospheric heights implies that the fluctuations could be of a different origin. Besides, the same slope at both low and high frequencies suggests that the entire spectrum could actually represent the universal signatures of SMAWs, which cause intensity fluctuations throughout the sunspot umbra. Furthermore, the good resemblance between these power spectra, and those generated from integrated disk light (Gabriel et al. 1997), confirms the source of sunspot oscillations in the quiet photosphere. The power enhancements near $5.5 \mathrm{mHz}$ highlights the predominance of three-minute oscillations in the umbral atmosphere.

We also computed phase difference spectra for different channel pairs, which suggest the oscillations with frequencies up to approximately $3 \mathrm{mHz}$ remain evanescent, while those with higher frequencies propagate universally upward. The theory of linear wave propagation in a stratified atmosphere, including aspects of radiative cooling (Centeno et al. 2006), agrees well with the phase spectra for the $\left(\mathrm{Mg} \mathrm{I}_{2}, \mathrm{Ca}\right.$ II K) and $\left(\mathrm{Mg} \mathrm{I}_{2}, \mathrm{H} \alpha\right)$ channel pairs. However, the phase spectra for the (Ca II K, H $\alpha$ ) and (IRIS $2796 \AA, 1330 \AA$ ) pairs indicate that either the individual channels in each pair form very closely $(<100 \mathrm{~km})$ to one another within the umbra, or that simple linear wave theory breaks down due to the predominantly nonlinear behavior of waves in these atmospheric regions.

The 3 minute $(\approx 5.5 \mathrm{mHz})$ oscillation amplitudes, as inferred from the normalized power, increase gradually up to the atmospheric height corresponding to the Ca II $\mathrm{K}$ observations, before subsequently decreasing beyond. The corresponding energy flux, however, appears to decrease steadily immediately upon leaving the photosphere. Recent observations of upwardly 
propagating slow magnetoacoustic sausage modes in a magnetic pore also reveal a gradual decrease in energy flux with height (Grant et al. 2015). As can be seen, a slightly steeper reduction in mechanical wave energy is observed above the Ca II K atmospheric height. This is qualitatively similar to the decay in acoustic energy flux calculated from three-dimensional MHD numerical simulations (Felipe et al. 2011). The authors describe the decay as being due to a combination of radiative losses and shock dissipation. The lower radiative cooling time in the chromosphere, together with the strong shock features found at $\mathrm{Ca}$ II $\mathrm{K}$ heights, leads to a steeper decrease in the energy flux at increasing atmospheric heights. Further studies including theoretical and numerical modeling might be useful to ascertain the role of any other damping mechanism(s).

Felipe et al. (2011) found an energy flux of the order of $10^{6} \mathrm{erg} \mathrm{cm}^{-2} \mathrm{~s}^{-1}$ at photospheric heights, and concluded that the energy flux available from acoustic oscillations is insufficient to balance the radiative losses in chromospheric umbrae. Recently, using simultaneous observations of a sunspot umbra with Hinode (Kosugi et al. 2007) and IRIS, Kanoh et al. (2016) estimated the energy fluxes for $6-10 \mathrm{mHz}$ SMAWs at the photospheric and lower transition region levels as $2 \times 10^{7} \mathrm{erg} \mathrm{cm}^{-2} \mathrm{~s}^{-1}$ and $8.3 \times 10^{4} \mathrm{erg} \mathrm{cm}^{-2} \mathrm{~s}^{-1}$, respectively. These results, in contrast to Felipe et al. (2011), demonstrate the potential for SMAWs to contribute significantly to the heating of the umbral chromosphere. The energy flux, in our results, decreases from about $1.3 \pm 0.1 \times$ $10^{7} \mathrm{erg} \mathrm{cm}^{-2} \mathrm{~s}^{-1}$ at the photosphere to about $40 \pm$ $3 \mathrm{erg} \mathrm{cm}^{-2} \mathrm{~s}^{-1}$ at the height corresponding to the IRIS $1330 \AA$ channel $(\sim 2000 \mathrm{~km})$. The latter value would be $765 \pm$ $57 \mathrm{erg} \mathrm{cm}^{-2} \mathrm{~s}^{-1}$ if the alternative height $(1500 \mathrm{~km})$, inferred from the phase difference spectra, is assumed for the IRIS $1330 \AA$ channel. In either case, these values indicate significant damping in agreement with Kanoh et al. (2016). Of course, it is not trivial to state whether or not the entire missing wave energy directly resulted in the thermalization of the local plasma. For instance, a good fraction of the wave energy could be transferred to other fast/Alfvén modes through the processes of mode conversion (e.g., Cally \& Goossens 2008; Cally \& Moradi 2013), resulting in dissipationless damping, which, in fact, can happen over several scale heights depending on the oscillation frequency. It is not possible to isolate this effect using the current observations.

The slope of the power spectra (power-law index) is found to increase with height. By comparing the velocity power spectra of propagating kink waves observed in the chromosphere and corona, Morton et al. (2014) demonstrated that there is enhanced damping at high frequencies as the waves propagate toward corona. It is possible that the SMAWs are displaying similar behavior, with stronger damping at higher frequencies, which results in steeper slopes at increased atmospheric heights. Indeed, the oscillation amplitudes above $\mathrm{Ca}$ II $\mathrm{K}$ height show frequency-dependent damping with shorter damping lengths for higher frequencies (see Figure 8). The greater radiative and/or conductive losses for high-frequency waves (Carlsson \& Stein 2002; Krishna Prasad et al. 2012) could perhaps be responsible for their stronger damping in these layers. Additionally, viscosity, ion-neutral collisions, ambipolar diffusion, and resonant absorption (via mode conversion) can also produce frequency-dependent damping with more efficiency at high frequencies.
Interestingly, the peak frequency of the 3 minute oscillations is found to shift from $5.65 \mathrm{mHz}(\approx 177 \mathrm{~s})$ at $\mathrm{Mg} \mathrm{I} \mathrm{b}_{2}$ heights to $6.57 \mathrm{mHz}(\approx 152 \mathrm{~s})$ at $\mathrm{Ca}$ II $\mathrm{K}$ heights, before remaining at that value for the other chromospheric and transition region channels. This is perhaps a consequence of the variation of acoustic cutoff frequency with height (Murawski et al. 2016; Wiśniewska et al. 2016). Wiśniewska et al. (2016) performed observations of the quiet solar atmosphere using multiple spectral lines and demonstrated that the acoustic cutoff frequency initially increases with height, before levelling off at greater atmospheric heights. In addition, the presence of harmonic peaks at 6.57 and $13.1 \mathrm{mHz}(152$ and $76 \mathrm{~s}$; see Figure 6) in the Ca II K and IRIS $2796 \AA$ channels may support the existence of a resonant cavity, possibly below the photosphere (Thomas \& Scheuer 1982), rather than in the chromosphere (Zhugzhda et al. 1983), since the oscillations are found to be predominantly upwardly propagating at chromospheric heights. This is in contrast to the results of Moreels et al. (2015), where the authors found standing slow modes in a magnetic pore supporting the chromospheric cavity.

Finally, a comparison of power spectra across the umbral radius reveals an abnormal enhancement in high-frequency $(>5.5 \mathrm{mHz}$ ) power close to the umbral barycenter in chromospheric channels. Although the change in magnetic field inclination angles across the umbra has been shown to produce similar effects (Reznikova et al. 2012; Madsen et al. 2015), the restriction of this behavior in our current observations to chromospheric channels (i.e., excluding the transition region observations) is puzzling and demands further exploration.

The authors thank the referee for useful comments. S.K.P. is grateful to the UK Science and Technology Facilities Council (STFC) for funding support that allowed this project to be undertaken. D.B.J. also wishes to thank the UK STFC for the award of an Ernest Rutherford Fellowship, in addition to a dedicated research grant. D.B.J. is also grateful to Invest NI and Randox Laboratories Ltd. for the award of a Research \& Development Grant (059RDEN-1). T.V.D. was supported by an Odysseus grant of the FWO Vlaanderen, the IAP P7/08 CHARM (Belspo) and the GOA-2015-014 (KU Leuven). This work was based on discussions at the ISSI and ISSI-Beijing. This project has received funding from the European Research Council (ERC) under the European Union's Horizon 2020 research and innovation programme (grant agreement No 724326). R.M. is grateful for the support of a Leverhulme Trust Early Career Fellowship. V.F. would like to thank the STFC and Royal Society-Newton Mobility Grant NI160149, for their financial support. R.E. is grateful to STFC (UK) and acknowledges The Royal Society (UK) for the support received. IRIS is a NASA small explorer mission developed and operated by LMSAL with mission operations executed at NASA Ames Research center and major contributions to downlink communications funded by ESA and the Norwegian Space Centre.

\section{ORCID iDs}

S. Krishna Prasad (iD https://orcid.org/0000-0002-0735-4501

D. B. Jess (iD https://orcid.org/0000-0002-9155-8039

T. Van Doorsselaere (iD https://orcid.org/0000-00019628-4113

G. Verth (i) https://orcid.org/0000-0002-9546-2368

R. J. Morton (i) https://orcid.org/0000-0001-5678-9002 
V. Fedun (1) https://orcid.org/0000-0002-0893-7346

R. Erdélyi (i) https://orcid.org/0000-0003-3439-4127

D. J. Christian (i) https://orcid.org/0000-0003-1746-3020

\section{References}

Abdelatif, T. E., Lites, B. W., \& Thomas, J. H. 1984, in Small-Scale Dynamical Processes in Quiet Stellar Atmospheres, ed. S. L. Keil (Sunspot, NM: National Solar Observatory), 141

Avrett, E. H. 1981, in The Physics of Sunspots, ed. L. E. Cram \& J. H. Thomas (Sunspot, NM: Sacramento Peak Observatory), 235

Beckers, J. M., \& Tallant, P. E. 1969, SoPh, 7, 351

Beebe, H. A., \& Johnson, H. R. 1969, SoPh, 10, 79

Bello González, N., Flores Soriano, M., Kneer, F., \& Okunev, O. 2009, A\&A, 508, 941

Bello González, N., Franz, M., Martínez Pillet, V., et al. 2010, ApJL, 723, L134

Berghmans, D., \& Clette, F. 1999, SoPh, 186, 207

Bloomfield, D. S., Lagg, A., \& Solanki, S. K. 2007, ApJ, 671, 1005

Cally, P. S. 2007, AN, 328, 286

Cally, P. S., Bogdan, T. J., \& Zweibel, E. G. 1994, ApJ, 437, 505

Cally, P. S., \& Goossens, M. 2008, SoPh, 251, 251

Cally, P. S., \& Moradi, H. 2013, MNRAS, 435, 2589

Carlsson, M., Hansteen, V. H., de Pontieu, B., et al. 2007, PASJ, 59, S663

Carlsson, M., \& Stein, R. F. 2002, in IAU Colloq. 188, Magnetic Coupling of the Solar Atmosphere, ed. H. Sawaya-Lacoste (ESA SP-505; Noordwijk: ESA), 293

Centeno, R., Collados, M., \& Trujillo Bueno, J. 2006, ApJ, 640, 1153

Centeno, R., Collados, M., \& Trujillo Bueno, J. 2009, ApJ, 692, 1211

Chae, J., Lee, J., Cho, K., et al. 2017, ApJ, 836, 18

De Moortel, I. 2009, SSRv, 149, 65

De Moortel, I., Ireland, J., \& Walsh, R. W. 2000, A\&A, 355, L23

De Pontieu, B., Erdélyi, R., \& De Moortel, I. 2005, ApJL, 624, L61

De Pontieu, B., Tarbell, T., \& Erdélyi, R. 2003, ApJ, 590, 502

De Pontieu, B., Title, A. M., Lemen, J. R., et al. 2014, SoPh, 289, 2733

Deubner, F.-L., \& Fleck, B. 1989, A\&A, 213, 423

Dorotovič, I., Erdélyi, R., Freij, N., Karlovský, V., \& Márquez, I. 2014, A\&A, 563, A12

Efron, B. 1979, AnSta, 7, 1

Erdélyi, R. 2006, in Proc. SOHO 18/GONG 2006/HELAS I, Beyond the Spherical Sun, ed. K. Fletcher \& M. Thompson (ESA SP-624; Noordwijk: ESA), 15

Fedun, V., Shelyag, S., \& Erdélyi, R. 2011, ApJ, 727, 17

Felipe, T., Khomenko, E., \& Collados, M. 2011, ApJ, 735, 65

Felipe, T., Khomenko, E., Collados, M., \& Beck, C. 2010, ApJ, 722, 131

Fleck, B., \& Schmitz, F. 1991, A\&A, 250, 235

Fossum, A., \& Carlsson, M. 2005, Natur, 435, 919

Freij, N., Dorotovič, I., Morton, R. J., et al. 2016, ApJ, 817, 44

Freij, N., Scullion, E. M., Nelson, C. J., et al. 2014, ApJ, 791, 61

Gabriel, A. H., Charra, J., Grec, G., et al. 1997, in IAU Symp. 181, Sounding Solar and Stellar Interiors, ed. J. Provost \& F.-X. Schmider (Dordrecht: Kluwer), 53

Giovanelli, R. G. 1972, SoPh, 27, 71

Grant, S. D. T., Jess, D. B., Moreels, M. G., et al. 2015, ApJ, 806, 132

Gupta, G. R. 2014, A\&A, 568, A96

Handy, B. N., Acton, L. W., Kankelborg, C. C., et al. 1999, SoPh, 187, 229

Jess, D. B., De Moortel, I., Mathioudakis, M., et al. 2012a, ApJ, 757, 160

Jess, D. B., Mathioudakis, M., Christian, D. J., et al. 2010, SoPh, 261, 363

Jess, D. B., Morton, R. J., Verth, G., et al. 2015, SSRv, 190, 103

Jess, D. B., Reznikova, V. E., Ryans, R. S. I., et al. 2016, NatPh, 12, 179

Jess, D. B., Reznikova, V. E., Van Doorsselaere, T., Keys, P. H., \& Mackay, D. H. 2013, ApJ, 779, 168

Jess, D. B., Shelyag, S., Mathioudakis, M., et al. 2012b, ApJ, 746, 183

Jess, D. B., Van Doorsselaere, T., Verth, G., et al. 2017, ApJ, 842, 59

Kanoh, R., Shimizu, T., \& Imada, S. 2016, ApJ, 831, 24

Khomenko, E., \& Cally, P. S. 2012, ApJ, 746, 68

Khomenko, E., Centeno, R., Collados, M., \& Trujillo Bueno, J. 2008, ApJL, 676, L85
Khomenko, E., \& Collados, M. 2015, LRSP, 12, 6

Kneer, F., Mattig, W., \& v. Uexkuell, M. 1981, A\&A, 102, 147

Kontogiannis, I., Tsiropoula, G., \& Tziotziou, K. 2010, A\&A, 510, A41

Kontogiannis, I., Tsiropoula, G., \& Tziotziou, K. 2014, A\&A, 567, A62

Kosugi, T., Matsuzaki, K., Sakao, T., et al. 2007, SoPh, 243, 3

Krishna Prasad, S., Banerjee, D., \& Gupta, G. R. 2011, A\&A, 528, L4

Krishna Prasad, S., Banerjee, D., \& Van Doorsselaere, T. 2014, ApJ, 789 118

Krishna Prasad, S., Banerjee, D., Van Doorsselaere, T., \& Singh, J. 2012, A\&A, 546, A50

Krishna Prasad, S., Jess, D. B., \& Khomenko, E. 2015, ApJL, 812, L15

Kuridze, D., Verth, G., Mathioudakis, M., et al. 2013, ApJ, 779, 82

Lawrence, J. K., Cadavid, A. C., Christian, D. J., Jess, D. B., \& Mathioudakis, M. 2011, ApJL, 743, L24

Lites, B. W., \& Thomas, J. H. 1985, ApJ, 294, 682

Madsen, C. A., Tian, H., \& DeLuca, E. E. 2015, ApJ, 800, 129

Maltby, P., Avrett, E. H., Carlsson, M., et al. 1986, ApJ, 306, 284

Mariska, J. T. 1993, The Solar Transition Region (Cambridge: Cambridge Univ. Press)

Markwardt, C. B. 2009, in ASP Conf. Ser. 411, Astronomical Data Analysis Software and Systems XVIII, ed. D. A. Bohlender, D. Durand, \& P. Dowler (San Francisco, CA: ASP), 251

McEwan, M. P., \& de Moortel, I. 2006, A\&A, 448, 763

Mihalas, D., \& Mihalas, B. W. 1984, Foundations of Radiation Hydrodynamics (New York: Oxford Univ. Press)

Moreels, M. G., Freij, N., Erdélyi, R., Van Doorsselaere, T., \& Verth, G. 2015, A\&A, 579, A73

Morton, R. J., Erdélyi, R., Jess, D. B., \& Mathioudakis, M. 2011, ApJL, 729, L18

Morton, R. J., Verth, G., Hillier, A., \& Erdélyi, R. 2014, ApJ, 784, 29

Morton, R. J., Verth, G., Jess, D. B., et al. 2012, NatCo, 3, 1315

Mumford, S. J., \& Erdélyi, R. 2015, MNRAS, 449, 1679

Mumford, S. J., Fedun, V., \& Erdélyi, R. 2015, ApJ, 799, 6

Murawski, K., Musielak, Z. E., Konkol, P., \& Wiśniewska, A. 2016, ApJ, 827, 37

Ofman, L., \& Wang, T. 2002, ApJL, 580, L85

Raja Bayanna, A., Mathew, S. K., Kumar, B., Louis, R. E., \& Venkatakrishnan, P. 2014, RAA, 14, 1458

Reznikova, V. E., Shibasaki, K., Sych, R. A., \& Nakariakov, V. M. 2012, ApJ, 746, 119

Rimmele, T. R. 2004, Proc. SPIE, 5490, 34

Rouppe van der Voort, L. H. M., Rutten, R. J., Sütterlin, P., Sloover, P. J., \& Krijger, J. M. 2003, A\&A, 403, 277

Schmieder, B. 1979, A\&A, 74, 273

Spruit, H. C. 1991, in Challenges to Theories of the Structure of Moderate Mass Stars, ed. D. O. Gough \& J. Toomre (Berlin: Springer), 121

Su, J. T. 2014, ApJ, 793, 117

Thomas, J. H., \& Weiss, N. O. (ed.) 1992, in Proc. of the NATO Advanced Research Workshop on the Theory of Sunspots, Sunspots: Theory and Observations, Vol. 375, ed. J. H. Thomas \& N. O. Weiss (Dordrecht: Kluwer), 3

Thomas, J. H., \& Scheuer, M. A. 1982, SoPh, 79, 19

Vecchio, A., Cauzzi, G., Reardon, K. P., Janssen, K., \& Rimmele, T. 2007, A\&A, 461, L1

Vernazza, J. E., Avrett, E. H., \& Loeser, R. 1981, ApJS, 45, 635

Vigeesh, G., Fedun, V., Hasan, S. S., \& Erdélyi, R. 2012, ApJ, 755, 18

Vigeesh, G., Hasan, S. S., \& Steiner, O. 2009, A\&A, 508, 951

Wang, T., Ofman, L., Sun, X., Provornikova, E., \& Davila, J. M. 2015, ApJL, 811, L13

Wedemeyer-Böhm, S., Steiner, O., Bruls, J., \& Rammacher, W. 2007, in ASP Conf. Ser. 368, The Physics of Chromospheric Plasmas, ed. P. Plasmas, I. Heinzel, \& R. J. Dorotovič (San Francisco, CA: ASP), 93

Wiśniewska, A., Musielak, Z. E., Staiger, J., \& Roth, M. 2016, ApJL, 819 L23

Withbroe, G. L., \& Noyes, R. W. 1977, ARA\&A, 15, 363

Wöger, F., von der Lühe, O., \& Reardon, K. 2008, A\&A, 488, 375

Zhao, J., Felipe, T., Chen, R., \& Khomenko, E. 2016, ApJL, 830, L17

Zhugzhda, I. D., Locans, V., \& Staude, J. 1983, SoPh, 82, 369 